TITLE:

\title{
Electronic excitation spectra of furan and pyrrole: Revisited by the symmetry adapted cluster- configuration interaction method
}

\section{$\operatorname{AUTHOR}(S)$ :}

Wan, J; Meller, J; Hada, M; Ehara, M; Nakatsuji, H

\section{CITATION:}

Wan, J ... [et al]. Electronic excitation spectra of furan and pyrrole: Revisited by the symmetry adapted cluster-configuration interaction method. JOURNAL OF CHEMICAL PHYSICS 2000, 113(18): 7853-7866

ISSUE DATE:

2000-11-08

URL:

http://hdl.handle.net/2433/50180

\section{RIGHT:}

Copyright 2000 American Institute of Physics. This article may be downloaded for personal use only. Any other use requires prior permission of the author and the American Institute of Physics. 


\title{
Electronic excitation spectra of furan and pyrrole: Revisited by the symmetry adapted cluster-configuration interaction method
}

\author{
Jian Wan, Jaroslaw Meller, Masahiko Hada, Masahiro Ehara, and Hiroshi Nakatsuji ${ }^{a}$ \\ Department of Synthetic Chemistry and Biological Chemistry, Graduate School of Engineering, \\ Kyoto University, Sakyo-ku, Kyoto 606-8501, Japan
}

(Received 20 June 2000; accepted 17 August 2000)

\begin{abstract}
Electronic excitation spectra of furan and pyrrole are reinvestigated by the symmetry-adapted cluster configuration-interaction method. The 47 and 46 lowest singlet and triplet electronic states are computed for furan and pyrrole, respectively. Two series $\left(1 a_{2}\right.$ and $\left.2 b_{1}\right)$ of low-lying Rydberg states and the valence $\pi-\pi^{*}$ excited states strongly influence each other in both furan and pyrrole. The present calculations give detailed and satisfactory theoretical assignments of the vacuum ultraviolet spectra and the electron energy-loss spectra of the two molecules. The similarities and differences in the electronic excitations between furan and pyrrole are discussed in detail. The accuracy and assignments of recent theoretical studies, i.e., complete active space second-order perturbation, multireference Møller-Plesset perturbation, second-order algebraic-diagrammatic construction, multireference double configuration interaction, and CC3, are compared. (C) 2000 American Institute of Physics. [S0021-9606(00)30842-X]
\end{abstract}

\section{INTRODUCTION}

Furan and pyrrole are so-called five-membered six $\pi$-electron aromatic ring molecules in which the heteroatoms oxygen and nitrogen donate two $\pi$ electrons and each of the four carbon atoms supplies one $\pi$ electron. The electronic spectra of furan and pyrrole have been the subjects of many experimental and theoretical studies. ${ }^{1-29}$ The interest in these molecules is not surprising, considering the importance of these molecules as fundamental units in many important biological molecules. Further, recently, the calculations of their electronic excitation spectra have been considered as benchmarks for theoretical studies of excited states. The representative theoretical studies have been carried out so far by Nakatsuji et al. ${ }^{21}$ in 1985, Serrano-Andres et al. ${ }^{22}$ in 1993 , Palmer and co-workers ${ }^{23,24}$ in 1995 and 1998, Trofimov and Schirmer ${ }^{26,27}$ in 1997, and Christiansen and co-workers ${ }^{28,29}$ in 1998 and 1999.

On the experimental side, ${ }^{1-15,23,24}$ three types of electronic excitation spectra were measured for furan and pyrrole: the vacuum ultraviolet (VUV) spectrum, electron energy-loss (EEL) spectrum, and ultraviolet photoelectron spectrum. These spectra provide information regarding singlet valence- and Rydberg-excited states, optically forbidden transitions such as triplet excitations, and ionization potentials, respectively.

On the other hand, the previous ab initio quantum theoretical investigations ${ }^{16-29}$ have demonstrated the underlying difficulties in calculating vertical excited states of furan and pyrrole. One is strong valence-Rydberg mixing. Calculations based only on the valence basis are obviously inadequate and this is a main reason for the failure of early semiempirical studies and early $a b$ initio studies. Second, the roles of electron correlations are crucial. Accurate and reliable results are

\footnotetext{
a) Author to whom correspondence should be addressed; electronic mail: hiroshi@sbchem.kyoto-u.ac.jp
}

obtained only by sophisticated electron-correlation methods for ground and excited states. Third, dynamic polarization of the $\sigma$ electrons occurs strongly for some electronic excitations. Fourth, all of the previous three factors interact rather strongly in furan and pyrrole.

As observed from the experimental excitation spectra of furan and pyrrole (Figs. 3 and 4), the peaks are broad and complicated for the existence of many valence- and Rydberg-excited states and their vibrational structures. Generally band maxima and vertical excitation energies differ substantially: even for Rydberg states the deviation exceeds $0.2 \mathrm{eV}$ in some cases. ${ }^{27}$ This point should be kept in mind when trying to do an assignment and judge the accuracy of calculations.

Among the previous ab initio theoretical studies on furan and pyrrole, the symmetry adapted cluster (SAC) and SACconfiguration interaction (SAC-CI) study ${ }^{21}$ was a pioneering work, but due to the computational resources available at that time, the rather limited basis sets and relatively small active space impaired the quality of the results. In the later complete active space second-order perturbation (CASPT2), ${ }^{22}$ multireference Møller-Plesset (MRMP) ${ }^{25}$ perturbation, second-order algebraic-diagrammatic construction $\operatorname{ADC}(2),{ }^{26,27}$ and a hierarchy of coupled cluster model (coupled-cluster single double, CC3) $^{28,29}$ studies, fairly large basis sets were used: the atomic natural orbital basis set contracted to $[4 s 3 p 1 d / 2 s 1 p]$ and augmented by ring-centered diffuse $s, p$, and $d$ functions in CASPT2, Dunning's ccpVTZ basis set augmented by charge-centered $3 s, 3 p$, and $3 d$ Rydberg functions in MRMP, cc-pVDZ augmented by atom-centered diffuse functions and molecule-centered diffuse functions in $\operatorname{ADC}(2)$, and cc-pVTZ augmented by atomcentered diffuse functions and molecule-centered diffuse functions in $\mathrm{CC} 3$.

However, there are still many inconsistencies among the results of these recent high-level theoretical studies with 


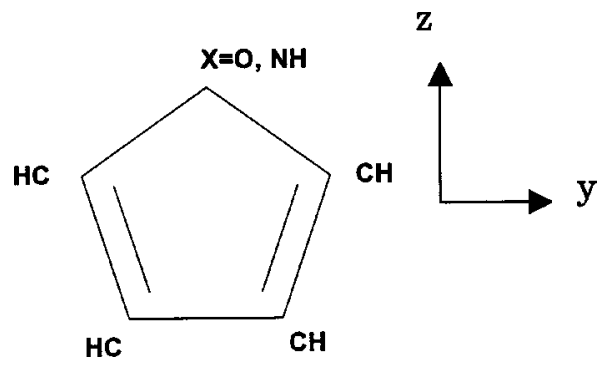

FIG. 1. Furan and pyrrole $\left(\mathrm{C}_{4} \mathrm{H}_{4} \mathrm{X}, \mathrm{X}=\mathrm{O}, \mathrm{NH}\right)$.

large basis sets. For example, the CASPT2 and MRMP methods, which use a complete active space self-consistent field reference function, gave very similar computational results for furan and proposed a relatively reasonable interpretation of the singlet valence excitations lying lower than 7.8 $\mathrm{eV}$, whereas, for pyrrole, they gave quite different results ( $0.51 \mathrm{eV}$ difference) for the lowest valence excitation of ${ }^{1} B_{2}$ symmetry, which was assigned to the first band of the VUV spectrum. For the same band, the $\mathrm{ADC}(2)$ studies made a conclusion that there was no valence excitation of ${ }^{1} B_{2}$ symmetry in this region, which was explained solely by the Rydberg transitions. Recently, the fourth $\pi-\pi^{*}$ valence transition, $V^{\prime}\left({ }^{1} B_{2}\right)$, was identified by Palmer et al. ${ }^{23}$ as an absorption peak at $8.7 \mathrm{eV}$ in the VUV spectrum of furan with the aid of multireference multiroot CI multireference double configuration interaction (MRDCI) calculations. CASPT2 calculated this state at $8.38 \mathrm{eV}$ with an intensity of 0.27 , and MRMP did not visit this region. The CC3 estimated the corresponding state at an energy region higher than $9 \mathrm{eV}$.

On the other hand, since pyrrole differs from furan only by the substitution of an $\mathrm{O}$ atom by a $\mathrm{N}-\mathrm{H}$ group, we would expect the electronic structures and excitation spectra of the two molecules to be similar. For example, the general profiles of VUV spectra of the two molecules ${ }^{23,24}$ are very similar in that they have two main intense and broad bands; the first is located at around $5.5-7 \mathrm{eV}$, and the second is more intense and located between 7.0 and $8.8 \mathrm{eV}$. However, an obvious difference exists in the spectra of pyrrole and furan, i.e., there is a weaker central band near $7 \mathrm{eV}$ in the spectrum of pyrrole. The similarities and differences in the electronic excitation spectra of furan and pyrrole, and the uncertainties in the assignments of the valence and Rydberg excitations in the spectra of furan and pyrrole have motivated us to reinvestigate them using the SAC/SAC-CI method. In Sec. II we outline the computational details. Calculated results and discussion are presented in Sec. III. A summary is given in Sec. IV.

\section{COMPUTATIONAL DETAILS}

The experimentally determined ground-state equilibrium geometries ${ }^{30}$ for furan and pyrrole with $C_{2 v}$ symmetry illustrated in Fig. 1 were commonly used for both ground and excited states, so that the calculated excitation energies are vertical in nature.

In the present calculations, we have used Dunning's augmented correlation consistent basis set AUG-cc-pVDZ ${ }^{31}$ for $\mathrm{O}, \mathrm{N}$ and $\mathrm{C}$ atoms, and cc-pVDZ ${ }^{32}$ for $\mathrm{H}$ atoms. For the molecule-centered Rydberg functions, we have used a set of diffuse functions $(3 s 3 p 3 d)$ selected from the studies of Kaufmann et al. ${ }^{33}$ and placed them on the molecular center of gravity. These are the same as those used by Trofimov and Schirmer. ${ }^{26,27}$ The total number of basis functions is 175 in furan and 180 in pyrrole. This type of augmented basis is believed to be adequate for describing widely different electronic structures of molecules in the ground state, singlet and triplet valence and Rydberg excited states, describing diffuse character and differential correlation effects. The number of Hartree-Fock canonical orbitals, calculated by the GAUSSIN98 package, ${ }^{34}$ is 175 , consisting of 18 occupied and 157 virtual orbitals in furan, and 180, consisting of 18 occupied and 162 virtual orbitals in pyrrole. The self-consistent field (SCF) occupied valence orbitals and some important virtual orbitals of furan and pyrrole are shown in Table I, respectively, together with their orbital energies, symmetries, and characters.

The details of the SAC/SAC-CI theory have been presented elsewhere. ${ }^{35-41}$ The SAC/SAC-CI calculations were done using the local version of the SAC-CI module. ${ }^{41}$ In all of the present SAC/SAC-CI calculations, the active space consists of 13 occupied orbitals and 157 and 162 virtual orbitals in furan and pyrrole, respectively: Only the $1 s$ core molecular orbitals are frozen. The details of the SAC/ SAC-CI calculations are exactly the same as those recently reported for cyclopentadiene. ${ }^{42}$

Table II shows the previous ${ }^{21}$ and present SAC/SAC-CI computational details on furan and pyrrole. Note that, 15 years ago, SAC/SAC-CI was the only sophisticated theory for calculating the molecular excited states. The computational resources were quite different from those available now: The available main memory, disk space, and computational time were very limited, although the previous calculations were certainly "big" by the standard of that time. But now, we can use a much better basis set and a much larger active space than those in the previous SAC/SAC-CI studies. This greatly improves the calculated results, even though the basic method used is the same, i.e., the SAC/SAC-CI method. Improvements in the SAC/SAC-CI program, algorithm, etc., are also important, but when we do the comparisons shown in Table II, we are strongly convinced that advances in computer technology over the past 15 years play the greatest role in the present improvement.

\section{RESULTS AND DISCUSSION}

In the four $\pi$-orbital approximation, the two occupied orbitals $2 b_{1}\left(\pi_{2}\right)$ and $1 a_{2}\left(\pi_{3}\right)$, and the two virtual orbitals $3 b_{1}\left(\pi_{4}^{*}\right)$ and $2 a_{2}\left(\pi_{5}^{*}\right)$ illustrated in Fig. 2 give rise to four singlet $\pi-\pi^{*}$ transitions: $\pi_{3} \rightarrow \pi^{*}(V)$ and $\pi_{2} \rightarrow \pi^{*}\left(V^{\prime}\right)$. These four transitions $V\left({ }^{1} B_{2}\right), V^{\prime}\left({ }^{1} A_{1}\right), V\left({ }^{1} A_{1}\right)$, and $V^{\prime}\left({ }^{1} B_{2}\right)$ are expected to lie below the first ionization potential $\left(\mathrm{IP}_{1}\right) 8.883 \mathrm{eV}$ in furan and $8.21 \mathrm{eV}$ in pyrrole, and would give absorption bands in the range of $5.5-8.8 \mathrm{eV}$.

In order to distinguish valence and Rydberg-type transitions, we use some properties discussed in our recent paper. ${ }^{42}$ First, we take advantage of the fact that the $A_{2}$ and $B_{1}$ Rydberg states are not perturbed by the valence $\pi-\pi^{*}$ 
TABLE I. Hartree-Fock occupied molecular orbitals (MOs) and some important unoccupied MOs of furan and pyrrole. $\sigma$ and $\pi$ stand for valence character and Ryd stands for Rydberg character.

\begin{tabular}{|c|c|c|c|c|c|c|}
\hline \multirow[b]{2}{*}{ MOs } & \multicolumn{2}{|c|}{ Symmetry } & \multicolumn{2}{|c|}{ Orbital energy $(\mathrm{eV})$} & \multicolumn{2}{|c|}{ Nature } \\
\hline & Furan & Pyrrole & Furan & Pyrrole & Furan & Pyrrole \\
\hline \multicolumn{7}{|l|}{ Occupied } \\
\hline Cores & & & & & $\sigma(1 s)$ & $\sigma(1 s)$ \\
\hline 6 & $4 a_{1}$ & $4 a_{1}$ & -39.820 & -35.263 & $\sigma(2 s)$ & $\sigma(2 s)$ \\
\hline 7 & $5 a_{1}$ & $5 a_{1}$ & -29.602 & -28.470 & $\sigma(2 s)$ & $\sigma(2 s)$ \\
\hline 8 & $3 b_{2}$ & $3 b_{2}$ & -27.409 & -26.621 & $\sigma(2 s)$ & $\sigma(2 s)$ \\
\hline 9 & $4 b_{2}$ & $6 a_{1}$ & -22.032 & -21.711 & $\sigma(2 s)$ & $\sigma(2 s)$ \\
\hline 10 & $6 a_{1}$ & $4 b_{2}$ & -21.314 & -20.913 & $\sigma(2 s)$ & $\sigma(2 s)$ \\
\hline 11 & $7 a_{1}$ & $7 a_{1}$ & -20.170 & -20.074 & $\sigma(2 p)$ & $\sigma(2 p)$ \\
\hline 12 & $1 b_{1}$ & $8 a_{1}$ & -17.230 & -16.181 & $\pi_{1}$ & $\sigma(2 p)$ \\
\hline 13 & $5 b_{2}$ & $5 b_{2}$ & -16.621 & -15.888 & $\sigma(2 p)$ & $\sigma(2 p)$ \\
\hline 14 & $6 b_{2}$ & $1 b_{1}$ & -15.694 & -15.430 & $\sigma(2 p)$ & $\pi_{1}$ \\
\hline 15 & $8 a_{1}$ & $6 b_{2}$ & -15.451 & -14.861 & $\sigma(2 p)$ & $\sigma(2 p)$ \\
\hline 16 & $9 a_{1}$ & $9 a_{1}$ & -14.732 & -14.411 & $\sigma(2 p)$ & $\sigma(2 p)$ \\
\hline 17 (next highest occupied molecular orbital) & $2 b_{1}$ & $2 b_{1}$ & -10.835 & -9.454 & $\pi_{2}$ & $\pi_{2}$ \\
\hline 18 (highest occupied molecular orbital) & $1 a_{2}$ & $1 a_{2}$ & -8.702 & -8.054 & $\pi_{3}$ & $\pi_{3}$ \\
\hline \multicolumn{7}{|l|}{ Unoccupied } \\
\hline 19 (lowest unoccupied molecular orbital) & $a_{1}$ & $a_{1}$ & 0.047 & 0.041 & $s$-Ryd & $s$-Ryd \\
\hline 20 & $b_{2}$ & $a_{1}$ & 0.162 & 0.146 & $p_{y}$-Ryd & $s / p_{z}$-Ryd \\
\hline 21 & $a_{1}$ & $b_{2}$ & 0.166 & 0.164 & $s / p_{z}-\mathrm{Ryd}$ & $p_{y}$-Ryd \\
\hline 23 & $a_{1}$ & $a_{1}$ & 0.201 & 0.207 & $s / d$-Ryd & $s / d$-Ryd \\
\hline 26 & $a_{2}$ & $b_{1}$ & 0.355 & 0.359 & $d_{x y}-$ Ryd & $d_{x z}-$ Ryd \\
\hline 27 & $a_{1}$ & $a_{2}$ & 0.360 & 0.360 & $s / d$-Ryd & $d_{x y}-\mathrm{Ryd}$ \\
\hline 29 & $a_{1}$ & $a_{1}$ & 0.484 & 0.447 & $s / d$-Ryd & $s / d_{y z}$-Ryd \\
\hline 30 & $b_{2}$ & $b_{2}$ & 0.544 & 0.553 & $p_{y}-\mathrm{Ryd}$ & $p_{y}-$ Ryd \\
\hline 32 & $b_{1}$ & $b_{1}$ & 0.589 & 0.596 & $p_{x}$-Ryd & $p_{x}$-Ryd \\
\hline 33 & $b_{2}$ & $a_{1}$ & 0.951 & 0.945 & $d_{y z}$-Ryd & $s / d$-Ryd \\
\hline 35 & $a_{1}$ & $a_{1}$ & 0.990 & 0.970 & $s / d$-Ryd & $s / d$-Ryd \\
\hline 36 & $a_{2}$ & $b_{1}$ & 1.002 & 1.012 & $d_{x y}-\mathrm{Ryd}$ & $d_{x z} / p_{x}-\mathrm{Ryd}$ \\
\hline 38 & $b_{1}$ & $a_{2}$ & 1.011 & 1.017 & $d_{x z} / p_{x}-\mathrm{Ryd}$ & $d_{x y}-$ Ryd \\
\hline 39 & $b_{2}$ & $a_{1}$ & 1.396 & 1.376 & $p_{y}$-Ryd & $s / p_{z}$-Ryd \\
\hline 40 & $a_{1}$ & $b_{2}$ & 1.432 & 1.423 & $s / p_{z}-\mathrm{Ryd}$ & $p_{y}$-Ryd \\
\hline 41 & $b_{1}$ & $b_{1}$ & 1.499 & 1.519 & $p_{x}$-Ryd & $p_{x}$-Ryd \\
\hline 42 & $a_{1}$ & $a_{1}$ & 1.874 & 1.876 & $s / d$-Ryd & $s / d$-Ryd \\
\hline 46 & $b_{1}$ & $b_{1}$ & 2.286 & 2.304 & $d_{x z} / p_{x}-\mathrm{Ryd}$ & $d_{x z} / p_{x}-\mathrm{Ryd}$ \\
\hline 48 & $b_{1}$ & $a_{1}$ & 3.439 & 3.436 & $\pi^{*}$ & $\sigma_{s} / d$-Ryd \\
\hline 50 & $b_{2}$ & $b_{2}$ & 3.587 & 3.666 & $p_{y}-\mathrm{Ryd}$ & $p_{y}$-Ryd \\
\hline 52 & $b_{2}$ & $b_{1}$ & 3.627 & 3.787 & $\sigma_{p y}$ & $\pi^{*}$ \\
\hline 53 & $a_{2}$ & $a_{2}$ & 4.043 & 4.145 & $\pi^{p y}$ & $\pi^{*}$ \\
\hline 57 & $b_{1}$ & $a_{1}$ & 4.837 & 4.920 & $\pi^{*}$ & $\sigma_{s} / d-\mathrm{Ryd}$ \\
\hline 59 & $a_{1}$ & $b_{1}$ & 4.909 & 5.149 & $\sigma_{s} / d-\operatorname{Ryd}$ & $\pi^{*}$ \\
\hline 61 & $b_{1}$ & $a_{2}$ & 6.298 & 6.054 & $\pi^{*}$ & $\pi^{*}$ \\
\hline 62 & $a_{1}$ & $b_{1}$ & 6.888 & 6.465 & $\sigma_{s}$ & $\pi^{*}$ \\
\hline 64 & $a_{2}$ & $b_{1}$ & 6.988 & 6.852 & $\pi^{*}$ & $\pi^{*}$ \\
\hline 66 & $b_{1}$ & $a_{2}$ & 8.258 & 7.254 & $\pi^{*}$ & $\pi^{*}$ \\
\hline
\end{tabular}

transitions due to the symmetry constraint. We then use the expectation values of the second moments of the charge distribution $\left\langle r^{2}\right\rangle$, and its components $\left\langle x^{2}\right\rangle,\left\langle y^{2}\right\rangle$, and $\left\langle z^{2}\right\rangle$. In addition, the oscillator strength has also been used by several authors as an indicator to compare valence and Rydberg characters. ${ }^{23,24,26,27}$ We will use all of them to analyze our SAC-CI results.

\section{A. Furan}

The calculated SCF energy of furan is -228.654184 hartrees. The SAC ground-state energy is -229.070173 hartrees, and the correlation energy is -0.415988 hartrees. The corresponding previous values were -228.55715 , -228.81055 , and -0.25340 hartrees, respectively.

The singlet vertical excitation energies calculated by the SAC-CI method are given in Table III, which also shows the oscillator strength $[f(r)]$ and the second moment of each excited state. In Table IV, the present SAC-CI singlet and triplet excitation energies are compared with the experimental values, and with the results obtained by CASPT2, MRDCI, ADC(2), and CC3. Since for furan the MRMP results are quite similar to those for CASPT2, they are not given in Table IV. Figure 3 shows a comparison of the experimental VUV spectrum $^{23}$ and the theoretical SAC-CI spectrum of furan. 
TABLE II. Comparison of the previous and present SAC/SAC-CI computational conditions for furan and pyrrole.

\begin{tabular}{|c|c|c|c|c|c|}
\hline & \multicolumn{3}{|c|}{ Previous (Ref. 6) } & \multicolumn{2}{|c|}{ Present } \\
\hline Basis sets & \multicolumn{3}{|c|}{$\begin{array}{l}\mathrm{DZ}+\text { Rydberg } \\
\mathrm{O}, \mathrm{N} \text { and } \mathrm{C}:[4 s 2 p] \\
\mathrm{H}:[2 s] \\
\text { Rydberg }(2 s 2 p 1 d) \text { on the } \\
\text { molecular center of gravity }\end{array}$} & \multicolumn{2}{|c|}{$\begin{array}{l}\text { AUG-cc-pVDZ+Rydberg } \\
\text { O, N and C: AUG-cc-pVDZ } \\
\text { H: cc-pVDZ } \\
\text { Rydberg }(3 s 3 p 3 d) \text { on the } \\
\text { molecular center of gravity }\end{array}$} \\
\hline $\begin{array}{l}\text { Active space } \\
\text { Furan } \\
\text { Pyrrole }\end{array}$ & \multicolumn{3}{|c|}{$67^{\mathrm{a}}\left[5^{\mathrm{b}}, 51^{\mathrm{c}}\right]$} & $\begin{array}{l}175^{\mathrm{a}}\left[5^{\mathrm{b}}, 170^{\mathrm{c}}\right] \\
180^{\mathrm{a}}\left[5^{\mathrm{b}} 175^{\mathrm{c}}\right]\end{array}$ & \\
\hline $\begin{array}{l}\text { Energy threshold } \\
\text { for perturbation } \\
\text { selection (PS) }\end{array}$ & \multicolumn{3}{|c|}{$\lambda_{g}=4.0 \times 10^{-5}, \lambda_{e}=5.0 \times 10^{-5}$} & \multicolumn{2}{|c|}{$\lambda_{g}=1.0 \times 10^{-5}, \lambda_{e}=1.0 \times 10^{-6}$} \\
\hline $\begin{array}{l}\text { Dimension of linked } \\
\text { operators after PS }\end{array}$ & \multicolumn{2}{|c|}{ Singlet } & Triplet & Singlet & Triplet \\
\hline \multirow{5}{*}{ Furan } & $A_{1}(G)^{\mathrm{d}}$ & 2456 & & 17010 & \\
\hline & $A_{1}$ & 1896 & 2496 & 61287 & 65942 \\
\hline & $A_{2}$ & 2260 & 2425 & 58006 & 69327 \\
\hline & $B_{1}$ & 2352 & 2017 & 106049 & 49416 \\
\hline & $B_{2}$ & 1805 & 2379 & 60292 & 59803 \\
\hline \multirow{5}{*}{ Pyrrole } & $A_{1}(G)^{\mathrm{d}}$ & 2457 & & 16437 & \\
\hline & $A_{1}$ & 1841 & 2515 & 67165 & 71068 \\
\hline & $A_{2}$ & 1883 & 2199 & 72433 & 88101 \\
\hline & $B_{1}$ & 1913 & 2487 & 89004 & 88690 \\
\hline & $B_{2}$ & 1758 & 2701 & 66404 & 65683 \\
\hline
\end{tabular}

${ }^{\mathrm{a}}$ The total number of MOs.

bThe number of frozen orbitals. 5 means " $1 s$ core",

${ }^{\mathrm{c}}$ The size of the active space, (occupied MO plus unoccupied MO).

${ }^{\mathrm{d}} \mathrm{SAC}$ ground state.

\section{Singlet valence excited states}

The first band system occurs in the region of $5.5-7.0 \mathrm{eV}$ of the VUV spectrum. The maximum of this band lies at 6.04 $\mathrm{eV}$ (peak I in Fig. 3), which was assigned to the valence $\pi-\pi^{*}$ transition $V\left({ }^{1} B_{2}\right)$ by Palmer et al. ${ }^{23}$ In addition, Flicker et $a l .^{8}$ observed the intensity maximum of a broad transition at $6.06 \mathrm{eV}$ using electron impact spectroscopy and assigned it to $\tilde{X}^{1} A_{1} \rightarrow{ }^{1} B_{2}$. In our present SAC-CI calculations, the excitation energy of the $1{ }^{1} B_{2}$ state is computed at $6.40 \mathrm{eV}$ with an oscillator strength of 0.1852 : it clearly represents the most intense feature of peak I. The second moment $\left\langle r^{2}\right\rangle$ is 92.5358 a.u. $^{2}$, which defines it as a valence excited state, even if it is somewhat more diffuse than the ground state $\left(\left\langle r^{2}\right\rangle=64.6624\right.$ a.u. $\left.{ }^{2}\right)$ due to valence-Rydberg mixing. The nature of the $1{ }^{1} B_{2}$ state is mainly the $\pi_{3}-\pi^{*}$ $(18 \rightarrow 48)$ excitations mixed with the Rydberg transitions $(18 \rightarrow 41),(18 \rightarrow 32)$, and $(18 \rightarrow 46)$, etc. We would like to assign the first peak at $6.06 \mathrm{eV}$ to the $1{ }^{1} B_{2}$ state. The exci-

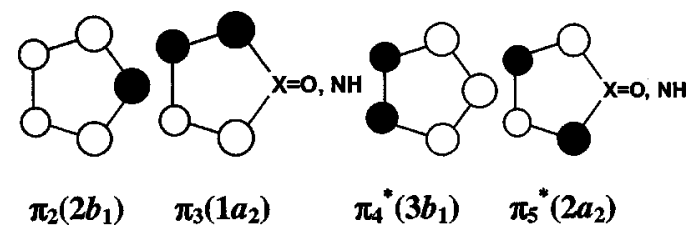

FIG. 2. Two highest occupied $\pi$ orbitals and two lowest unoccupied $\pi^{*}$ orbitals of furan and pyrrole. tation energies of the $1{ }^{1} B_{2}$ state were calculated to be 6.04 , 6.76, 5.95, 6.37, and $6.35 \mathrm{eV}$ by CASPT2, MRDCI, MRMP, $\mathrm{ADC}(2)$, and $\mathrm{CC} 3$, respectively. The corresponding oscillator strengths are $0.1543,0.183,0.158,0.148$, and 0.144 , respectively. When we estimate the accuracy of these theoretical results, we should take into account the difference between the vertical excitation energy and the peak maximum. For example, it has been reported ${ }^{28}$ that the difference between the vertical and the maximum peak excitation energies is at least $0.11 \mathrm{eV}$ for the ${ }^{1} B_{2 u}$ valence excited state of benzene.

The region of greatest intensity in the VUV spectrum occurs from approximately 7.2 to $8.4 \mathrm{eV}$, with an intensity maximum at $7.8 \mathrm{eV}$ (peak II in Fig. 3), to which the valence transition $V\left({ }^{1} A_{1}\right)$ has been assigned by most experimental and theoretical studies. Our present results calculated the $6{ }^{1} A_{1}$ state to lie at $8.34 \mathrm{eV}$ above the ground state with an intensity of 0.4826 . It is clearly related to the most intense feature of peak II. Its second moment $\left\langle r^{2}\right\rangle$ is 97.5047 a.u. ${ }^{2}$, which shows that it is a valence excited state, although it is somewhat more diffuse than the ground state due to slight valence-Rydberg mixing. The nature of the $6{ }^{1} A_{1}$ state is a mixing of $\pi_{3}-\pi^{*}(18 \rightarrow 53)$ and $\pi_{2}-\pi^{*}(17 \rightarrow 48)$ excitations and the Rydberg transitions $(17 \rightarrow 46)$ and $(17 \rightarrow 38)$, etc. Based on these results, we assign the $6{ }^{1} A_{1}$ state as the main component of the $V\left({ }^{1} A_{1}\right)$ transition. Note that the nature of the $6{ }^{1} A_{1}$ state is mixing of $\pi_{3}-\pi^{*}$ and $\pi_{2}-\pi^{*}$ 
TABLE III. Calculated excitation energies (in eV), second moments, and main configurations by the SAC/ SAC-CI method for furan.

\begin{tabular}{|c|c|c|c|c|c|c|c|c|}
\hline \multirow[b]{2}{*}{ State } & \multirow[b]{2}{*}{ Nature $^{a}$} & \multirow[b]{2}{*}{$\Delta E^{\mathrm{b}}$} & \multirow[b]{2}{*}{$\mathrm{Pol}^{\mathrm{c}}$} & \multirow[b]{2}{*}{$f(r)$} & \multicolumn{4}{|c|}{ Second moment } \\
\hline & & & & & $\left\langle x^{2}\right\rangle$ & $\left\langle y^{2}\right\rangle$ & $\left\langle z^{2}\right\rangle$ & $\left\langle r^{2}\right\rangle$ \\
\hline $1{ }^{1} A_{1}$ & Ground & 0.0 & & 0 & 24.6628 & 18.4772 & 21.5224 & 64.6624 \\
\hline $1{ }^{1} A_{2}$ & $3 s-R$ & 5.99 & & Forbid & 42.5477 & 34.8039 & 38.6635 & 116.0150 \\
\hline $1{ }^{1} B_{2}$ & $V$-mix & 6.40 & $y$ & 0.1852 & 42.7817 & 23.8893 & 25.8647 & 92.5358 \\
\hline $1{ }^{1} B_{1}$ & $3 p_{y}-R$ & 6.45 & $x$ & 0.0313 & 37.0420 & 65.4676 & 34.1141 & 136.6238 \\
\hline $2{ }^{1} A_{2}$ & $3 p_{z}-R$ & 6.66 & & Forbid & 40.3748 & 33.2618 & 75.0431 & 148.6797 \\
\hline $2{ }^{1} A_{1}$ & $V$ & 6.79 & $z$ & 0.0000 & 25.9881 & 20.0661 & 22.9068 & 68.9609 \\
\hline $2{ }^{1} B_{2}$ & $3 p_{x}-R$ & 6.82 & $y$ & 0.0158 & 65.3598 & 26.3912 & 37.7410 & 129.4920 \\
\hline $3{ }^{1} A_{2}$ & $3 d-R$ & 7.04 & & Forbid & 41.6107 & 68.7722 & 33.1252 & 143.5080 \\
\hline $2{ }^{1} B_{1}$ & $3 d_{y z}-R$ & 7.14 & $x$ & 0.0005 & 37.0320 & 60.9984 & 64.9757 & 163.0060 \\
\hline $4{ }^{1} A_{2}$ & $3 d-R$ & 7.27 & & Forbid & 52.6200 & 30.0711 & 89.0683 & 171.7594 \\
\hline $3{ }^{1} A_{1}$ & $3 d_{x y}-R$ & 7.36 & $z$ & 0.0001 & 91.1382 & 80.6382 & 40.2160 & 211.9923 \\
\hline $3{ }^{1} B_{1}$ & $3 s^{\prime}-R$ & 7.45 & $x$ & 0.0292 & 45.5458 & 47.8471 & 45.2990 & 138.6919 \\
\hline $5{ }^{1} A_{2}$ & $4 s-R$ & 7.50 & & Forbid & 153.8467 & 90.5160 & 99.9118 & 344.2746 \\
\hline $3{ }^{1} B_{2}$ & $3 d_{x z}-R$ & 7.51 & $y$ & 0.0194 & 108.2377 & 40.0669 & 97.4018 & 245.7064 \\
\hline $4{ }^{1} B_{1}$ & $4 p_{y}-R$ & 7.67 & $x$ & 0.0008 & 94.2931 & 226.6794 & 90.7120 & 411.6845 \\
\hline $4{ }^{1} B_{2}$ & $4 p_{x}-R$ & 7.71 & $y$ & 0.0010 & 246.8240 & 86.1320 & 96.7509 & 429.7070 \\
\hline $6{ }^{1} A_{2}$ & $4 p_{z}-R$ & 7.72 & & Forbid & 104.4467 & 91.4211 & 255.1376 & 451.0054 \\
\hline $7{ }^{1} A_{2}$ & $4 d-R$ & 7.85 & & Forbid & 113.9521 & 256.0963 & 75.7213 & 445.7697 \\
\hline $5{ }^{1} B_{1}$ & $4 d_{y z}-R$ & 7.89 & $x$ & 0.0000 & 79.7302 & 185.1881 & 190.2943 & 455.2126 \\
\hline $8{ }^{1} A_{2}$ & $4 d-R$ & 7.93 & & Forbid & 121.5102 & 76.7263 & 269.3098 & 467.5463 \\
\hline $4{ }^{1} A_{1}$ & $4 d_{x y}-R$ & 7.99 & $z$ & 0.0002 & 211.5385 & 200.0038 & 80.1687 & 491.7110 \\
\hline $9{ }^{1} A_{2}$ & $5 s-R$ & 8.04 & & Forbid & 366.5189 & 246.8423 & 260.1989 & 873.5601 \\
\hline $5{ }^{1} B_{2}$ & $4 d_{x z}-R$ & 8.07 & $y$ & 0.0069 & 225.2252 & 78.9295 & 209.5113 & 513.6660 \\
\hline $6{ }^{1} B_{1}$ & $3 p_{z}^{\prime}-R$ & 8.07 & $x$ & 0.0006 & 44.3096 & 48.8139 & 78.7930 & 171.9166 \\
\hline $5{ }^{1} A_{1}$ & $3 p_{x}^{\prime}-R$ & 8.14 & $z$ & 0.0205 & 83.4876 & 39.7525 & 37.0449 & 160.2850 \\
\hline $7{ }^{1} B_{1}$ & $5 p_{y}-R$ & 8.18 & $x$ & 0.0023 & 145.3949 & 381.7329 & 142.6457 & 669.7735 \\
\hline $6{ }^{1} B_{2}$ & $5 p_{x}-R$ & 8.19 & $y$ & 0.0016 & 389.8390 & 133.7052 & 144.3590 & 667.9031 \\
\hline $10{ }^{1} A_{2}$ & $5 p_{z}-R$ & 8.21 & & Forbid & 147.3133 & 136.8059 & 392.3236 & 676.4428 \\
\hline $6{ }^{1} A_{1}$ & $V$-mix & 8.34 & $z$ & 0.4826 & 38.4469 & 26.9042 & 32.1536 & 97.5047 \\
\hline $8{ }^{1} B_{1}$ & $5 d_{y z}-R$ & 8.47 & $x$ & 0.0017 & 54.9059 & 120.4527 & 100.1038 & 275.4624 \\
\hline $9{ }^{1} B_{1}$ & $3 d^{\prime}-R$ & 8.54 & $x$ & 0.0095 & 62.5576 & 135.8883 & 117.9192 & 316.3651 \\
\hline $10{ }^{1} B_{1}$ & $3 d^{\prime}-R$ & 8.67 & $x$ & 0.0292 & 57.9288 & 47.8102 & 74.4203 & 180.1593 \\
\hline $7{ }^{1} A_{1}$ & $5 d_{x y}-R$ & 8.69 & $z$ & 0.0035 & 184.2435 & 173.1875 & 71.3490 & 428.7800 \\
\hline $7{ }^{1} B_{2}$ & $5 d_{x z}-R$ & 8.75 & $y$ & 0.0222 & 168.8308 & 63.9795 & 157.0494 & 389.8597 \\
\hline $8{ }^{1} B_{2}$ & $3 d_{x y}^{\prime}-R$ & 8.79 & $y$ & 0.0243 & 88.9270 & 68.6441 & 50.3966 & 207.9677 \\
\hline $11^{1} B_{1}$ & $3 d^{\prime}-R$ & 8.87 & $x$ & 0.0048 & 38.4421 & 52.1882 & 72.9737 & 163.6040 \\
\hline $8{ }^{1} A_{1}$ & $4 s^{\prime}-R$ & 8.95 & $x$ & 0.0036 & 135.2292 & 98.2036 & 116.8031 & 350.2359 \\
\hline $12{ }^{1} B_{1}$ & $3 d_{x z}^{\prime}-R$ & 8.95 & $z$ & 0.0703 & 104.0758 & 41.4103 & 97.6589 & 243.1450 \\
\hline $9{ }^{1} B_{2}$ & $V$-mix & 9.08 & $y$ & 0.1662 & 56.3822 & 41.8921 & 32.1471 & 130.4213 \\
\hline $13{ }^{1} B_{1}$ & $4^{\prime} p_{z}-R$ & 9.15 & $x$ & 0.0003 & 111.3931 & 93.3319 & 239.8221 & 444.5471 \\
\hline $1{ }^{3} B_{2}$ & $V$ & 4.39 & & $\cdots$ & 25.0331 & 18.3365 & 22.6064 & 65.9760 \\
\hline $1{ }^{3} A_{1}$ & $V$ & 5.63 & & $\cdots$ & 25.2181 & 19.2344 & 22.5002 & 66.9527 \\
\hline $1{ }^{3} A_{2}$ & $3 s-R$ & 5.98 & & $\cdots$ & 43.0576 & 34.1056 & 37.2876 & 114.4508 \\
\hline $1^{3} B_{1}$ & $3 p_{y}-R$ & 6.52 & & $\cdots$ & 37.8233 & 67.2748 & 34.9733 & 140.0714 \\
\hline $2^{3} B_{2}$ & $3 p_{x}-R$ & 6.66 & & $\cdots$ & 73.5041 & 29.5236 & 34.6416 & 137.6693 \\
\hline $2^{3} A_{2}$ & $3 p_{z} / s-R$ & 6.68 & & $\cdots$ & 39.7699 & 33.0344 & 74.0609 & 146.8652 \\
\hline $2^{3} A_{1}$ & $V$ & 7.10 & & $\cdots$ & 29.0880 & 22.2528 & 23.5868 & 74.9276 \\
\hline $2^{3} B_{1}$ & $3 d_{y z}-R$ & 7.21 & & $\cdots$ & 38.2497 & 64.9995 & 68.9878 & 172.2370 \\
\hline
\end{tabular}

${ }^{\mathrm{a}} V$ and $R$ denote, respectively, valence and Rydberg excited state. For details see the text.

${ }^{\mathrm{b}}$ Transition energy.

${ }^{\mathrm{c}}$ Polarization direction of the transition moment.

transitions, and the notation $V\left({ }^{1} A_{1}\right)$, based the simple four $\pi$-orbital model, is inadequate because " $V$ " denotes only $\pi_{3}-\pi^{*}$ type transitions.

CASPT2 computed the $4{ }^{1} A_{1}$ state at $7.74 \mathrm{eV}$ with an intensity of 0.4159 , and it was reported ${ }^{21}$ that the appearance of intruder states in CASPT2 wave function renders an uncertainty of about $0.2 \mathrm{eV}$ to this state. MRDCI computed this state at $8.324 \mathrm{eV}$ with an intensity of 0.476. MRMP com- puted the $4{ }^{1} A_{1}$ state at $7.69 \mathrm{eV}$ with an oscillator strength of $0.494, \operatorname{ADC}(2)$ at $8.16 \mathrm{eV}$ with an oscillator strength of $0.308, \mathrm{CC} 3$ at $8.35 \mathrm{eV}$ with an oscillator strength of 0.350 .

Recently, Palmer et $a l .{ }^{23}$ assigned an absorption peak at $8.7 \mathrm{eV}$ in their VUV spectrum (peak III in Fig. 3) to the valence excitation $V^{\prime}\left({ }^{1} B_{2}\right)$ with the aid of MRDCI calculated results: $9.422 \mathrm{eV}$ with an intensity of 0.197 . In their studies, the relative intensities were important for relating 
TABLE IV. SAC-CI results compared with the experimental excitation energies and other theoretical results for furan.

\begin{tabular}{|c|c|c|c|c|c|c|c|c|c|c|c|c|}
\hline \multirow[b]{2}{*}{ State } & \multirow[b]{2}{*}{ Nature $^{a}$} & \multicolumn{2}{|c|}{$\mathrm{SAC}-\mathrm{CI}$} & \multirow[b]{2}{*}{ Expt. $^{\mathrm{c}}$} & \multicolumn{2}{|c|}{ CASPT $^{d}$} & \multicolumn{2}{|c|}{$\mathrm{CC} 3(\mathrm{CCSD})^{\mathrm{e}}$} & \multicolumn{2}{|c|}{$\mathrm{MRDCI}^{\mathrm{g}}$} & \multicolumn{2}{|c|}{$\operatorname{ADC}(2)^{\mathrm{i}}$} \\
\hline & & $f(r)$ & $\Delta E^{\mathrm{b}}$ & & $\Delta E$ & State & $\Delta E^{\mathrm{f}}$ & Nature & $\Delta E^{\mathrm{h}}$ & Nature & $\Delta E$ & Nature \\
\hline $1^{1} A_{2}$ & $3 s-R$ & Forbid & 5.99 & 5.91 & 5.92 & $1^{1} A_{2}$ & 6.11 & $3 s\left({ }^{1} A_{2}\right)$ & 5.950 & $3 s\left({ }^{1} A_{2}\right)$ & 5.86 & $3 s\left({ }^{1} A_{2}\right)$ \\
\hline $1{ }^{1} B_{2}$ & $V$ & 0.1852 & 6.40 & 6.04 & 6.04 & $1{ }^{1} B_{2}$ & 6.35 & $V\left({ }^{1} B_{2}\right)$ & 6.759 & $V\left({ }^{1} B_{2}\right)$ & 6.37 & $V\left({ }^{1} B_{2}\right)$ \\
\hline $1{ }^{1} B_{1}$ & $3 p_{y}-R$ & 0.0313 & 6.45 & 6.47 & 6.46 & $1{ }^{1} B_{1}$ & 6.64 & $3 p_{y}\left({ }^{1} B_{1}\right)$ & 6.659 & $3 p_{x}\left({ }^{1} B_{2}\right)$ & 6.35 & $3 p\left({ }^{1} B_{1}\right)$ \\
\hline $2{ }^{1} A_{2}$ & $3 p_{z}-R$ & Forbid & 6.66 & 6.61 & 6.59 & $2{ }^{1} A_{2}$ & 6.80 & $3 p_{z}\left({ }^{1} A_{2}\right)$ & & & 6.50 & $3 p\left({ }^{1} A_{2}\right)$ \\
\hline $2{ }^{1} A_{1}$ & $V$ & 0.0000 & 6.79 & & 6.16 & $2{ }^{1} A_{1}$ & 6.61 & $V\left({ }^{1} A_{1}\right)$ & 6.024 & $V\left({ }^{1} A_{1}\right)$ & 6.70 & $V^{\prime}\left({ }^{1} A_{1}\right)$ \\
\hline $2{ }^{1} B_{2}$ & $3 p_{x}-R$ & 0.0158 & 6.82 & 6.75 & 6.48 & $2{ }^{1} B_{2}$ & 6.94 & $3 p_{x}\left({ }^{1} B_{2}\right)$ & 6.633 & $3 p_{y}\left({ }^{1} B_{1}\right)$ & 6.73 & $3 p\left({ }^{1} B_{2}\right)$ \\
\hline $3{ }^{1} A_{2}$ & $3 d-R$ & Forbid & 7.04 & & 7.00 & $3{ }^{1} A_{2}$ & 7.12 & $3 d_{z}^{2}\left({ }^{1} A_{2}\right)$ & & & & \\
\hline $2{ }^{1} B_{1}$ & $3 d_{y z}-R$ & 0.0005 & 7.14 & & 7.15 & $2{ }^{1} B_{1}$ & & & & & 6.89 & $3 d\left({ }^{1} A_{2}\right)$ \\
\hline $4{ }^{1} A_{2}$ & $3 d-R$ & Forbid & 7.27 & & 7.22 & $4{ }^{1} A_{2}$ & & & & & 6.98 & $3 d\left({ }^{1} B_{1}\right)$ \\
\hline $3{ }^{1} A_{1}$ & $3 d_{x y}-R$ & 0.0001 & 7.36 & 7.28 & 7.31 & $3{ }^{1} A_{1}$ & 7.32 & $3 d_{y z}\left({ }^{1} B_{1}\right)$ & 6.988 & $3 d_{y z}\left({ }^{1} B_{1}\right)$ & 7.22 & $3 d\left({ }^{1} A_{1}\right)$ \\
\hline $3{ }^{1} B_{1}$ & $3 s^{\prime}-R$ & 0.0292 & 7.45 & 7.38 & 7.21 & $3{ }^{1} B_{1}$ & 7.52 & $3 s^{\prime}\left({ }^{1} B_{1}\right)$ & 7.143 & $3 s^{\prime}\left({ }^{1} B_{1}\right)$ & 7.05 & $3 s^{\prime}\left({ }^{1} B_{1}\right)$ \\
\hline $5{ }^{1} A_{2}$ & $4 s-R$ & Forbid & 7.50 & & & & & & & & 7.80 & $4 s\left({ }^{1} A_{2}\right)$ \\
\hline $3{ }^{1} B_{2}$ & $3 d_{x z}-R$ & 0.0194 & 7.51 & 7.43 & 7.13 & $3{ }^{1} B_{2}$ & 7.58 & $3 d_{x y}\left({ }^{1} A_{1}\right)$ & 7.405 & $3 d_{z}^{2}\left({ }^{1} A_{2}\right)$ & 7.11 & $3 d\left({ }^{1} A_{2}\right)$ \\
\hline $4{ }^{1} B_{1}$ & $4 p_{y}-R$ & 0.0008 & 7.67 & 7.52 & & & 7.70 & $4 s\left({ }^{1} A_{2}\right)$ & 7.594 & $4 s\left({ }^{1} A_{2}\right)$ & 7.30 & $4 s\left({ }^{1} A_{2}\right)$ \\
\hline $4{ }^{1} B_{2}$ & $4 p_{x}-R$ & 0.0010 & 7.71 & 7.53 & & & 7.72 & $3 d_{x z}\left({ }^{1} B_{2}\right)$ & 7.750 & $3 d_{x y}\left({ }^{1} A_{1}\right)$ & 7.35 & $3 d\left({ }^{1} B_{2}\right)$ \\
\hline $6{ }^{1} A_{2}$ & $4 p_{z}-R$ & Forbid & 7.72 & & & & & & & & & \\
\hline $7^{1} A_{2}$ & $4 d-R$ & Forbid & 7.85 & 7.70 & & & & & 7.824 & $4 p_{x}\left({ }^{1} B_{2}\right)$ & 7.48 & $4 p\left({ }^{1} B_{1}\right)$ \\
\hline $5{ }^{1} B_{1}$ & $4 d_{y z}-R$ & 0.0000 & 7.89 & & & & 7.90 & $4 p_{y}\left({ }^{1} B_{1}\right)$ & & & & \\
\hline $8{ }^{1} A_{2}$ & $4 d-R$ & Forbid & 7.93 & 7.79 & & & 7.94 & $4 p_{x}\left({ }^{1} B_{2}\right)$ & 7.652 & $4 p_{y}\left({ }^{1} B_{2}\right)$ & 7.53 & $4 p\left({ }^{1} B_{2}\right)$ \\
\hline $4{ }^{1} A_{1}$ & $4 d_{x y}-R$ & 0.0002 & 7.99 & & & & & & & & 7.51 & $4 p\left({ }^{1} A_{2}\right)$ \\
\hline $9{ }^{1} A_{2}$ & $5 s-R$ & Forbid & 8.04 & & & & & & & & 7.57 & $3 P^{\prime}\left({ }^{1} A_{2}\right)$ \\
\hline & & & & & & & & & & & 7.72 & $4 d\left({ }^{1} B_{1}\right)$ \\
\hline & & & & & & & & & & & 7.87 & $4 d\left({ }^{1} B_{2}\right)$ \\
\hline $5{ }^{1} B_{2}$ & $4 d_{x z}-R$ & 0.0069 & 8.07 & 8.01 & & & 8.26 & $4 d_{x z}\left({ }^{1} B_{2}\right)$ & & & 7.82 & $4 d\left({ }^{1} A_{1}\right)$ \\
\hline $6{ }^{1} B_{1}$ & $3 p_{z}^{\prime}-R$ & 0.0006 & 8.07 & 8.04 & & & 8.20 & $4 d_{x y}\left({ }^{1} A_{1}\right)$ & 7.563 & $4 d_{z}^{2}\left({ }^{1} A_{2}\right)$ & 7.66 & $4 d\left({ }^{1} A_{2}\right)$ \\
\hline & & & & & & & & & 8.581 & $4 d_{x y}\left({ }^{1} A_{1}\right)$ & 7.72 & $4 d\left({ }^{1} A_{2}\right)$ \\
\hline & & & & & & & & & 8.844 & $5 s\left({ }^{1} A_{2}\right)$ & 7.80 & $5 s\left({ }^{1} A_{2}\right)$ \\
\hline $5{ }^{1} A_{1}$ & $3 p_{x}^{\prime}-R$ & 0.0205 & 8.14 & 8.10 & & & 8.26 & $3 p_{x}^{\prime}\left({ }^{1} A_{1}\right)$ & 8.148 & $3 p_{x}^{\prime}\left({ }^{1} A_{1}\right)$ & 7.61 & $3 p^{\prime}\left({ }^{1} B_{1}\right)$ \\
\hline $7{ }^{1} B_{1}$ & $5 p_{y}-R$ & 0.0023 & 8.18 & 8.19 & & & 8.34 & $5 p_{y}\left({ }^{1} B_{1}\right)$ & 8.040 & $3 p_{z}^{\prime}\left({ }^{1} B_{1}\right)$ & 7.96 & $5 p\left({ }^{1} B_{1}\right)$ \\
\hline $6{ }^{1} B_{2}$ & $5 p_{x}-R$ & 0.0016 & 8.19 & 8.23 & & & 8.36 & $5 p_{x}\left({ }^{1} B_{2}\right)$ & & & 7.99 & $5 p\left({ }^{1} B_{2}\right)$ \\
\hline $10{ }^{1} \mathrm{~A}_{2}$ & $5 p_{z}-R$ & Forbid & 8.21 & & & & & & & & 7.71 & $3 p^{\prime}\left({ }^{1} A_{1}\right)$ \\
\hline $6{ }^{1} A_{1}$ & $V$-mix & 0.4826 & 8.34 & 7.8 & 7.74 & $4{ }^{1} A_{1}$ & 8.35 & $V\left({ }^{1} A_{1}\right)$ & 8.324 & $V\left({ }^{1} A_{1}\right)$ & 8.16 & $V\left({ }^{1} A_{1}\right)$ \\
\hline $8{ }^{1} B_{1}$ & $5 d_{y z}-R$ & 0.0017 & 8.47 & 8.32 & & & 8.52 & $5 d_{x z}\left({ }^{1} B_{2}\right)$ & & & & \\
\hline $9{ }^{1} B_{1}$ & $3 d^{\prime}-R$ & 0.0095 & 8.54 & 8.34 & & & 8.50 & $5 d_{x y}\left({ }^{1} A_{1}\right)$ & & & 8.06 & $3 d^{\prime}\left({ }^{1} B_{1}\right)$ \\
\hline $10{ }^{1} B_{1}$ & $3 d_{y z}^{\prime}-R$ & 0.0292 & 8.67 & 8.46 & & & & & 8.327 & $3 d_{x z}^{\prime}\left({ }^{1} A_{1}\right)$ & 8.32 & $3 d^{\prime}\left({ }^{1} B_{2}\right)$ \\
\hline $7^{1} A_{1}$ & $5 d_{x y}-R$ & 0.0035 & 8.69 & & & & & & 8.359 & $3 d_{x-y}^{\prime 22}\left({ }^{1} B_{1}\right)$ & 8.23 & $3 d^{\prime}\left({ }^{1} B_{1}\right)$ \\
\hline $7^{1} B_{2}$ & $5 d_{x z}-R$ & 0.0222 & 8.75 & & & & & & & & & \\
\hline $8^{1} B_{2}$ & $3 d_{x y}^{\prime}-R$ & 0.0243 & 8.79 & 8.77 & & & & & 8.936 & $3 d_{x y}^{\prime}-\left({ }^{1} B_{2}\right)$ & 8.51 & $3 d^{\prime}\left({ }^{1} A_{1}\right)$ \\
\hline $11^{1} B_{1}$ & $3 d^{\prime}-R$ & 0.0048 & 8.87 & & & & & & 8.395 & $3 d_{z}^{\prime 2}\left({ }^{1} B_{1}\right)$ & & \\
\hline $8^{1} A_{1}$ & $3 d_{x z}^{\prime}-R$ & 0.0036 & 8.95 & & & & & & & & & \\
\hline $12{ }^{1} B_{1}$ & $4 s^{\prime}-R$ & 0.0703 & 8.95 & & & & & & & & & \\
\hline $9{ }^{1} B_{2}$ & $V$-mix & 0.1662 & 9.08 & 8.7 & 8.38 & $V^{1} B_{2}$ & $>9$ & $V\left({ }^{1} B_{2}\right)$ & 9.422 & $V^{\prime}\left({ }^{1} B_{2}\right)$ & 8.64 & $V^{\prime}\left({ }^{1} B_{2}\right)$ \\
\hline $13{ }^{1} B_{1}$ & $4^{\prime} p_{z}-R$ & 0.0003 & 9.15 & 8.96 & & & & & & & 8.41 & $4 s^{\prime}\left({ }^{1} B_{1}\right)$ \\
\hline $1{ }^{3} B_{2}$ & $V$ & $\cdots$ & 4.39 & 3.99 & 3.99 & & & & 3.931 & & 4.31 & \\
\hline $1{ }^{3} A_{1}$ & V & $\cdots$ & 5.63 & 5.15 & 5.15 & & & & 5.282 & & 5.50 & \\
\hline $1^{3} A_{2}$ & $3 s-R$ & $\cdots$ & 5.98 & & 5.86 & & & & 8.682 & & 5.81 & \\
\hline $1{ }^{3} B_{1}$ & $3 p_{y}-R$ & $\cdots$ & 6.52 & & 6.42 & & & & 9.077 & & 6.31 & \\
\hline $2{ }^{3} B_{2}$ & $3 p_{x}-R$ & $\cdots$ & 6.66 & $\sim 6.5$ & & & & & 6.313 & & & \\
\hline $2{ }^{3} A_{2}$ & $3 p_{z} / s-R$ & $\cdots$ & 6.68 & & & & & & 8.733 & & & \\
\hline $2{ }^{3} A_{1}$ & $V$ & $\cdots$ & 7.10 & $\sim 6.5$ & & & & & 6.648 & & & \\
\hline
\end{tabular}

${ }^{\mathrm{a}} V$ and $R$ denote, respectively, valence and Rydberg excited state. For details see the text.

${ }^{\mathrm{b}}$ Transition energy (in eV).

${ }^{\mathrm{c}}$ Experimental data were taken from Refs. $21-25$ and references therein.

${ }^{\mathrm{d}}$ Reference 21.

${ }^{\mathrm{e}}$ Reference 25.

${ }^{\mathrm{f}} \mathrm{CC} 3$ for valence and CCSD for Rydberg.

${ }^{g}$ Reference 22.

${ }^{\mathrm{h}}$ Different basis sets were used for valence and Rydberg.

${ }^{\mathrm{i}}$ Reference 24 . 


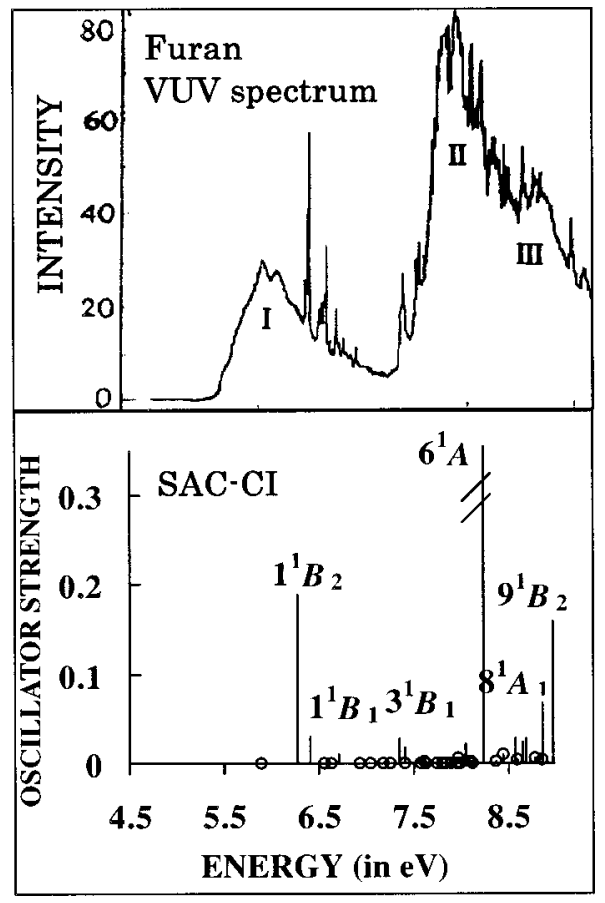

FIG. 3. VUV spectrum and SAC-CI theoretical spectrum of furan.

calculated results with experimental data when MRDCI results did not agree with the experimental data for the higher $\pi-\pi^{*}$ transitions. Our present results give the $9{ }^{1} B_{2}$ state at $9.08 \mathrm{eV}$ with an intensity of 0.1662 in this region. The second moment $\left\langle r^{2}\right\rangle$ of this state is 130.4213 a.u. ${ }^{2}$, which clearly shows that this state has significant diffuse character. Its nature is mainly due to the $\pi_{2}-\pi^{*} \quad(17 \rightarrow 53)$ and $(17 \rightarrow 64)$ excitations $V^{\prime}\left({ }^{1} B_{2}\right)$ mixed by the Rydberg transitions $(17 \rightarrow 26)$ and $(17 \rightarrow 36)$, etc. CASPT2 calculated this state at $8.38 \mathrm{eV}$ with an intensity of 0.27. MRMP did not give this state. $\mathrm{ADC}(2)$ computed this state at $8.64 \mathrm{eV}$ with an oscillator strength of 0.116 . CC3 estimated this state to be above $9 \mathrm{eV}$ and therefore outside of the energy range considered.

Finally, we discuss the valence excitation, $V^{\prime}\left({ }^{1} A_{1}\right)$, which is expected to occur in the first band. However, contrary to the $V\left({ }^{1} B_{2}\right)$ valence excitation which has been relatively well established, the $V^{\prime}\left({ }^{1} A_{1}\right)$ excitation is so complicated that it has not yet been reasonably assigned. There is a progression of weak peaks at 5.70,5.80,5.89, and $5.91 \mathrm{eV}$ in the UV absorption spectrum. This was assigned to a Rydberg transition and its vibronic lines by Robin, ${ }^{15}$ and to the $V^{\prime}\left({ }^{1} A_{1}\right)$ valence transition by Roebber et al. ${ }^{13}$ Palmer et al. stated that their recent work ${ }^{23}$ supported the valence-type assignment because their MRDCI calculations gave the energy order ${ }^{1} A_{1}<{ }^{1} B_{2}$ for the two lowest valence transitions, although the numerical results for these two states changed greatly with different basis sets. The ${ }^{1} A_{1}$ state was calculated at $6.024 \mathrm{eV}$ with an intensity of 0.011 , and the ${ }^{1} B_{2}$ state was calculated at $6.759 \mathrm{eV}$ with an intensity of 0.183 using the double zeta plus polarization (DZP) basis, and these two states were calculated at 6.63 and $6.88 \mathrm{eV}$, respectively, using the DZPR basis in a MRDCI study. ${ }^{23}$ Note that the nature of the $2{ }^{1} A_{1}$ state in the MRDCI study is only $\pi_{2} \rightarrow \pi^{*}$.
CASPT2 computed the $2{ }^{1} A_{1}$ state at $6.16 \mathrm{eV}$ with an intensity of 0.0015 , and its nature was reported to be a mixture of the configurations $2 b_{1} \rightarrow 3 b_{1}$ and $1 a_{2} \rightarrow 2 a_{2}$ and the double excitation of $\left[\left(1 a_{2}\right)^{2} \rightarrow\left(3 b_{1}\right)^{2}\right]$. MRMP computed the $2{ }^{1} A_{1}$ state at $6.16 \mathrm{eV}$ with an intensity of 0.0035 , and its nature was almost the same as that by CASPT2, Rydberg mixing was also found. $\mathrm{ADC}(2)$ computed this state at $6.70 \mathrm{eV}$ with an oscillator strength of 0.003 . CC3 computed it to be 6.61 $\mathrm{eV}$ with an oscillator strength of 0.000 . Our present calculations give the $2{ }^{1} A_{1}$ state at $6.79 \mathrm{eV}$ with an oscillator strength of 0.0000 . Its second moment $\left\langle r^{2}\right\rangle$ is 68.9609 a.u. ${ }^{2}$, which clearly defines it as a valence excited state, and its nature is a mixture of $\pi_{2}-\pi^{*}(17 \rightarrow 48)$ and $(17 \rightarrow 61)$ and $\pi_{3}-\pi^{*}$ transitions $(18 \rightarrow 53)$ and $(18 \rightarrow 64)$. Double excitation makes a rather small contribution. Note that all of the above-mentioned theoretical methods, except MRDCI, gave an energy order of ${ }^{1} B_{2}<{ }^{1} A_{1}$. This implies that the first ${ }^{1} A_{1}$ valence state should be located at a higher energy shoulder of the first band. Due to the very weak intensity of this valence transition, there is no obvious experimental evidence to confirm the theoretical results, and vice versa. However, in the case of thiophene, which will be discussed in a later paper, ${ }^{43}$ the $2{ }^{1} A_{1}$ state has been well characterized with regard to both excitation energy and oscillator strength, in comparison with the experimental data of the VUV and magnetic circular dichroism spectra.

\section{Singlet Rydberg states}

The lowest singlet Rydberg transition has been assigned to the $1 a_{2} \rightarrow 3 s$ Rydberg series. Robin ${ }^{15}$ first assigned the weak feature at $5.94 \mathrm{eV}$ to the dipole-forbidden ${ }^{1} A_{2}$ Rydberg state, and later Roebber et $a l^{13}$ also assigned the weak peak at $5.91 \mathrm{eV}$ to the $1 a_{2} \rightarrow 3 \mathrm{~s}$ Rydberg state using multiphoton ionization spectroscopy (MPI). In the present calculations, the $1{ }^{1} A_{2}$ state is computed at $5.99 \mathrm{eV}$ and its nature is a $3 s$-Rydberg transition. The CASPT2, MRDCI, MRMP, ADC(2), and CCSD methods computed this state at 5.92, $5.95,5.84,5.86$, and $6.11 \mathrm{eV}$, respectively.

The group of transitions in the region $6.4-6.8 \mathrm{eV}$ has usually been assigned to the $1 a_{2} \rightarrow 3 p$ Rydberg series. Derrick and co-workers ${ }^{5,6}$ assigned the band with a $0-0$ origin at $6.475 \mathrm{eV}$ to the transition $1 a_{2} \rightarrow 3 p b_{2}$. Cooper et al. ${ }^{12}$ assigned $6.47 \mathrm{eV}$ to the $3 p$ Rydberg states in their MPI spectroscopy studies, which supported the results of Derrick and co-workers. Recently, the $3 p b_{2}\left({ }^{1} B_{1}\right)$ assignment was questioned by Palmer et al., ${ }^{22}$ who assigned $6.472 \mathrm{eV}$ to the ${ }^{1} B_{2}$ state on the basis of their MRDCI calculations. Our present calculations computed the $2{ }^{1} B_{2}$ state at $6.82 \mathrm{eV}$, well separated from the $1{ }^{1} B_{1}$ transition at $6.45 \mathrm{eV}$, which is consistent with the $\mathrm{ADC}(2)$ and CCSD results. Therefore, we assigned an observed transition at $6.47 \mathrm{eV}$ to the $1 a_{2}$ $\rightarrow 3 p_{y}\left(1^{1} B_{1}\right)$ Rydberg series.

The nature of the transition observed at $6.75 \mathrm{eV}$ has been the subject of controversy. ${ }^{12,14,23}$ Cooper et al. ${ }^{12}$ assigned it tentatively to the ${ }^{1} A_{1}$ valence-type transition. This was criticized by Robin, ${ }^{15}$ and more recently, this peak at $6.75 \mathrm{eV}$ was assigned to the $3 p$-Rydberg transition by Nyulaszi. ${ }^{14}$ Recently, Palmer et al. ${ }^{23}$ convincingly proved the $3 p$-Rydberg origin of the $6.75 \mathrm{eV}$ band, but assigned it to 
$1 a_{2} \rightarrow 3 p_{y}\left({ }^{1} B_{1}\right)$ based on their MRDCI results. Taking into account the discussion on the $6.47 \mathrm{eV}$ transition, we believe the $2{ }^{1} B_{2}$ state is a better candidate for the observed $6.75 \mathrm{eV}$ transition, which is consistent with the ADC(2) and CCSD results. CASPT2 computed the $1{ }^{1} B_{1}$ and $2{ }^{1} B_{2}$ states at 6.46 and $6.48 \mathrm{eV}$, respectively, and assigned both of them to the $6.47 \mathrm{eV}$ transition. MRMP computed the $1{ }^{1} B_{1}$ and $2{ }^{1} B_{2}$ state at 6.40 and $6.50 \mathrm{eV}$, respectively, and also assigned both of them to the $6.47 \mathrm{eV}$ transition. Apparently, the observed $6.75 \mathrm{eV}$ transition was overlooked by the CASPT2 and MRMP studies, which is rather misleading because no evidence for a second transition at $6.48 \mathrm{eV}$ can be found in the experimental spectrum. In addition, we computed the dipole-forbidden $2{ }^{1} A_{2}$ state at $6.66 \mathrm{eV}$, which is a $1 a_{2}$ $\rightarrow 3 p_{z}$ Rydberg series.

Following the above-mentioned three $3 p$-Rydberg states, there is an experimentally identified Rydberg transition at $7.28 \mathrm{eV}$. This transition was interpreted as the $3 d$-Rydberg series, ${ }^{5,7,14}$ however, the spatial symmetry designation of this series is again not fully secure. Derrick and co-workers ${ }^{5,6}$ experimentally assigned the transition at 7.28 $\mathrm{eV}$ to the $3{ }^{1} A_{1}$ state. CASPT2 calculations gave the $2{ }^{1} B_{1}$ state at $7.15 \mathrm{eV}$ and the $3{ }^{1} A_{1}$ state at $7.31 \mathrm{eV}$, and followed the assignment of Derrick and co-workers MRMP and ADC(2) also followed the choice of CASPT2: the $2{ }^{1} B_{1}$ and $3{ }^{1} A_{1}$ states were computed at 7.10 and $7.26 \mathrm{eV}$ by MRMP, and at 6.98 and $7.22 \mathrm{eV}$ by $\mathrm{ADC}(2)$, respectively. Recently, however, Palmer et al. ${ }^{22}$ assigned the observed $7.28 \mathrm{eV}$ transition to the $3 d\left({ }^{1} B_{1}\right)$ state according to their MRDCI results: the $2{ }^{1} B_{1}$ state at $6.988 \mathrm{eV}$ and the $3{ }^{1} A_{1}$ state at $7.75 \mathrm{eV}$. CCSD calculated the $3 d_{y z}\left({ }^{1} B_{1}\right)$ state at $7.32 \mathrm{eV}$ and followed the assignment by Palmer et al. Our present results computed the $2{ }^{1} B_{1}\left(3 d_{y z}\right)$ state at $7.14 \mathrm{eV}$, and the $3{ }^{1} A_{1}\left(3 d_{x y}\right)$ state at $7.36 \mathrm{eV}$. The assignment to the $3 d_{x y}\left({ }^{1} A_{1}\right)$ state seems more natural, and therefore we assign the $7.28 \mathrm{eV}$ transition to the $3{ }^{1} A_{1}\left(3 d_{x y}\right)$ Rydberg series, as with CASPT2, MRMP, and $\operatorname{ADC}(2)$.

The next transition at $7.38 \mathrm{eV}$ has usually been assigned to the $1 b_{1} \rightarrow 3 s$ Rydberg series $\left(3 s^{\prime}\right) .^{5,12,23}$ Our present calculations gave the $3{ }^{1} B_{1}\left(3 s^{\prime}\right)$ state at $7.45 \mathrm{eV}$. CASPT2, MRDCI, MRMP, ADC(2), and CCSD calculations gave this state at 7.21, 7.143, 7.31, 7.05, and $7.52 \mathrm{eV}$, respectively.

Regarding the transitions at 7.43, 7.52, and $7.53 \mathrm{eV}$, MRDCI calculations assigned them to the dipole-forbidden transitions $1 a_{2} \rightarrow 3 d\left({ }^{1} A_{2}, 7.405 \mathrm{eV}\right), 1 a_{2} \rightarrow 4 s\left({ }^{1} A_{2}, 7.594\right.$ $\mathrm{eV})$ and the dipole-allowed transition $1 a_{2} \rightarrow 3 d\left({ }^{1} A_{1}, 7.750\right.$ $\mathrm{eV})$ states, respectively. $\mathrm{ADC}(2)$ studies assigned these three transitions to the $3 d\left({ }^{1} A_{2}, 7.22 \mathrm{eV}\right), 4 s\left({ }^{1} A_{2}, 7.30 \mathrm{eV}\right)$, and $3 d\left({ }^{1} B_{2}, 7.35 \mathrm{eV}\right)$ states, respectively. However, according to our present results, we prefer to tentatively assign them to $3{ }^{1} B_{2}\left(3 d_{x z}, 7.51 \mathrm{eV}\right), 4{ }^{1} B_{1}\left(4 p_{y}, 7.67 \mathrm{eV}\right)$, and $4{ }^{1} B_{2}$ $\left(4 p_{x}, 7.71 \mathrm{eV}\right)$. Our present assignment of Rydberg transitions lower than $7.53 \mathrm{eV}$ is consistent with the energy order and gap of the experimental data.

In the energy region higher than $7.53 \mathrm{eV}$, Rydberg transitions become increasingly dense, and the assignment of individual Rydberg transitions is more difficult. Therefore, the assignments of the experimental data higher than $7.53 \mathrm{eV}$ given in Table IV are tentative.

\section{Triplet excited states}

The two lowest triplet transitions of furan have been well identified by electron energy-loss (EEL) spectroscopy ${ }^{8-10,23}$ at 3.99 and $5.2 \mathrm{eV}$, which are assigned to the ${ }^{3} B_{2}$ and ${ }^{3} A_{1}$ valence transitions, respectively. Our present calculations computed the $1{ }^{3} B_{2}$ state at $4.39 \mathrm{eV}$ with a second moment of 65.9760 a.u. ${ }^{2}$, and $1^{3} A_{1}$ at $5.63 \mathrm{eV}$ with a second moment of 66.9527 a.u. ${ }^{2}$. The second moments of these two states clearly characterize them as valence excitations. The main configurations of ${ }^{3} B_{2}$ are $(18 \rightarrow 48)$, $(18 \rightarrow 61),(18 \rightarrow 57)$, etc., and those of ${ }^{3} A_{1}$ are $(17 \rightarrow 48)$, $(18 \rightarrow 64),(18 \rightarrow 53),(17 \rightarrow 61)$, etc.

Recently, Palmer et al. ${ }^{23}$ observed two higher triplet transitions at around $6.5 \mathrm{eV}$ in their EEL spectrum study and assigned them to the $2{ }^{3} A_{1}$ and $2{ }^{3} B_{2}$ states. Our present calculations gave the $3 p$ series of triplet Rydberg states in this energy region, $1{ }^{3} B_{1}\left(3 p_{y}\right)$ at $6.52 \mathrm{eV}, 2{ }^{3} B_{2}\left(3 p_{x}\right)$ at $6.66 \mathrm{eV}$, and $2^{3} A_{2}\left(3 p_{z}\right)$ at $6.68 \mathrm{eV}$. The valence triplet excited state $2{ }^{3} A_{1}$ was given at $7.10 \mathrm{eV}$ with the main configurations of $(17 \rightarrow 48),(18 \rightarrow 53), \quad(18 \rightarrow 64)$, etc. These states are related to the two higher triplet transitions in the EEL spectrum.

\section{B. Pyrrole}

The SCF energy of pyrrole is -208.838068 hartrees. The SAC ground-state energy is -209.218937 hartrees, and the correlation energy is -0.380869 hartrees. The corresponding previous values were $-208.75472,-208.99336$, and -0.23864 hartrees, respectively.

The principal SAC-CI results for the singlet and triplet vertical excitation energies are shown in Table $\mathrm{V}$, along with the oscillator strength $[f(r)]$ and second moment of each excited state. In Table VI, the present SAC-CI singlet and triplet excitation energies are compared to the experimental values, and to the results obtained by CASPT2, MRMP, $\mathrm{ADC}(2)$, and CC3. Figure 4 shows a comparison of the experimental VUV spectrum ${ }^{24}$ and the theoretical SAC-CI spectrum of pyrrole. Additionally, to interpret the similarities and differences between the VUV spectra of pyrrole and furan, the excitation energies, oscillator strengths, second moments, and main configurations of the valence excitations of the two molecules are compared in Table VII.

\section{The first intense and broad band (5.2-6.6 eV) of the VUV spectrum}

Our present calculations show that the first three ${ }^{1} B_{2}$ states are computed at 5.88, 6.48, and $6.76 \mathrm{eV}$, respectively, with oscillator strengths of $0.0805,0.0475$ and 0.0789 , respectively. The second moments of the three excited states are 132.1, 146.3, and 224.7 a.u. $^{2}$, respectively. It is difficult to distinguish these three states as valence excitation or Rydberg excitation by the second moments and oscillator strengths. However, we can use a simple hybrid model to give an interpretation. Since there are three configurations ${ }^{1}{ }^{1} B_{2}\left(1 a_{2} \rightarrow 3 p_{x}\right), \quad 2{ }^{1} B_{2} \quad$ (valence $1 a_{2} \rightarrow \pi^{*}$ ), and $3{ }^{1} B_{2}\left(1 a_{2} \rightarrow 3 d_{x z}\right)$ which are close in energy, we can assume that these three configurations $\left(1 a_{2} \rightarrow 3 p_{x}, \pi^{*}\right.$, and $\left.3 d_{x z}\right)$ are strongly mixed with each other and then construct three new hybrid excited states $1{ }^{1} B_{2}\left(1 a_{2}\right.$ 
TABLE V. Calculated excitation energies (in eV), oscillator strengths, and second moments of pyrrole.

\begin{tabular}{|c|c|c|c|c|c|c|c|c|}
\hline \multirow[b]{2}{*}{ State } & \multirow[b]{2}{*}{ Nature $^{a}$} & \multirow[b]{2}{*}{$\Delta E^{\mathrm{b}}$} & \multirow[b]{2}{*}{$\mathrm{Pol}^{\mathrm{c}}$} & \multirow[b]{2}{*}{$f(r)$} & \multicolumn{4}{|c|}{ Second moment } \\
\hline & & & & & $\left\langle x^{2}\right\rangle$ & $\left\langle y^{2}\right\rangle$ & $\left\langle z^{2}\right\rangle$ & $\left\langle r^{2}\right\rangle$ \\
\hline $1{ }^{1} A_{1}$ & Ground & 0 & & 0 & 26.3093 & 20.6830 & 18.3334 & 65.3256 \\
\hline $1{ }^{1} A_{2}$ & $3 s-R$ & 5.11 & & Forbid & 38.5385 & 31.6834 & 44.4993 & 114.7213 \\
\hline $1{ }^{1} B_{1}$ & $3 p_{y}-R$ & 5.80 & $x$ & 0.0211 & 40.1805 & 69.3496 & 35.1595 & 144.6895 \\
\hline $2{ }^{1} A_{2}$ & $3 p_{z}-R$ & 5.81 & & Forbid & 44.0000 & 38.9442 & 56.7104 & 139.6547 \\
\hline $1{ }^{1} B_{2}$ & $3 p_{x}-R$ & 5.88 & $y$ & 0.0805 & 70.6606 & 31.5707 & 29.9041 & 132.1354 \\
\hline $2{ }^{1} B_{1}$ & $3 s^{\prime}-R$ & 6.05 & $x$ & 0.0005 & 39.1957 & 37.2409 & 43.7521 & 120.1888 \\
\hline $3{ }^{1} A_{2}$ & $3 d-R$ & 6.38 & & Forbid & 42.1899 & 78.4625 & 47.3118 & 167.9642 \\
\hline $3{ }^{1} B_{1}$ & $3 d_{y z}-R$ & 6.39 & $x$ & 0.0037 & 40.0846 & 64.5531 & 64.3982 & 169.0359 \\
\hline $2{ }^{1} A_{1}$ & $V$ & 6.41 & $z$ & 0.0002 & 29.5900 & 24.3270 & 19.4281 & 73.3451 \\
\hline $4{ }^{1} A_{2}$ & $3 d-R$ & 6.44 & & Forbid & 70.1989 & 31.9981 & 57.5656 & 159.7626 \\
\hline $2{ }^{1} B_{2}$ & $V-3 d_{x z}-R$ & 6.48 & $y$ & 0.0475 & 68.3278 & 30.0456 & 47.9410 & 146.3143 \\
\hline $3{ }^{1} A_{1}$ & $3 d_{x y}-R$ & 6.64 & $z$ & 0.0000 & 95.7588 & 83.8498 & 37.4214 & 217.0300 \\
\hline $4{ }^{1} B_{1}$ & $3 p_{z}^{\prime}-R$ & 6.68 & $x$ & 0.0266 & 44.3956 & 44.7269 & 61.0966 & 150.2190 \\
\hline $5{ }^{1} A_{2}$ & $4 s-R$ & 6.71 & & Forbid & 112.8202 & 79.8926 & 147.4559 & 340.1687 \\
\hline $3{ }^{1} B_{2}$ & $3 d_{x z}-R$ & 6.76 & $y$ & 0.0789 & 103.2229 & 41.5147 & 79.9981 & 224.7356 \\
\hline $4{ }^{1} A_{1}$ & $3 p_{x}^{\prime}-R$ & 6.86 & $z$ & 0.0146 & 81.7840 & 39.3594 & 32.4668 & 153.6102 \\
\hline $5{ }^{1} B_{1}$ & $4 p_{y}-R$ & 6.93 & $x$ & 0.0029 & 103.5279 & 254.7510 & 99.6378 & 457.9166 \\
\hline $6{ }^{1} A_{2}$ & $4 p_{z}-R$ & 6.95 & & Forbid & 131.9602 & 107.6015 & 177.5677 & 417.1295 \\
\hline $4{ }^{1} B_{2}$ & $4 p_{x}-R$ & 6.99 & $y$ & 0.0020 & 244.7481 & 87.0448 & 89.8109 & 421.6038 \\
\hline $7{ }^{1} A_{2}$ & $4 d-R$ & 7.11 & & Forbid & 167.1016 & 189.0867 & 88.1108 & 444.2990 \\
\hline $8{ }^{1} A_{2}$ & $4 d-R$ & 7.13 & & Forbid & 105.1136 & 149.2357 & 231.5695 & 485.9187 \\
\hline $6{ }^{1} B_{1}$ & $4 d_{y z}-R$ & 7.14 & $x$ & 0.0001 & 86.5404 & 203.5861 & 194.1834 & 484.3100 \\
\hline $5{ }^{1} A_{1}$ & $4 d_{x y}-R$ & 7.26 & $z$ & 0.0041 & 216.4420 & 205.8745 & 77.9965 & 500.3131 \\
\hline $7{ }^{1} B_{1}$ & $3 d^{\prime}-R$ & 7.26 & $x$ & 0.0018 & 49.2432 & 59.8176 & 59.5719 & 168.6327 \\
\hline $5{ }^{1} B_{2}$ & $4 d_{x z}-R$ & 7.26 & $y$ & 0.0119 & 209.6781 & 75.2864 & 199.8960 & 484.8605 \\
\hline $9{ }^{1} A_{2}$ & $5 s-R$ & 7.27 & & Forbid & 296.8269 & 230.0584 & 330.6280 & 857.5133 \\
\hline $8{ }^{1} B_{1}$ & $3 d^{\prime}-R$ & 7.34 & $x$ & 0.0092 & 63.3648 & 73.0189 & 33.5406 & 169.9242 \\
\hline $10{ }^{1} A_{2}$ & $5 p_{z}-R$ & 7.44 & & Forbid & 195.3911 & 177.9971 & 371.4336 & 744.8217 \\
\hline $9{ }^{1} B_{1}$ & $5 p_{y}-R$ & 7.44 & $x$ & 0.0048 & 142.7447 & 373.8345 & 138.7340 & 655.3133 \\
\hline $6{ }^{1} B_{2}$ & $5 p_{x}-R$ & 7.45 & $y$ & 0.0020 & 390.0070 & 135.3600 & 136.3607 & 661.7277 \\
\hline $6{ }^{1} A_{1}$ & $3 d_{x z}^{\prime}-R$ & 7.49 & $z$ & 0.0679 & 88.5856 & 41.1360 & 73.4847 & 203.2063 \\
\hline $7{ }^{1} B_{2}$ & $3 d_{x y}^{\prime}-R$ & 7.55 & $y$ & 0.0052 & 100.2877 & 91.3482 & 37.2567 & 228.8926 \\
\hline $10{ }^{1} B_{1}$ & $4 s^{\prime}-R$ & 7.63 & $x$ & 0.0000 & 115.5165 & 80.3642 & 149.7550 & 345.6358 \\
\hline $11{ }^{1} B_{1}$ & $5 d_{y z}-R$ & 7.75 & $x$ & 0.0002 & 83.3076 & 190.2939 & 187.5531 & 461.1546 \\
\hline $7{ }^{1} A_{1}$ & $4^{\prime} p_{x}-R$ & 7.83 & $z$ & 0.0020 & 243.7424 & 93.9043 & 89.3948 & 427.0415 \\
\hline $8{ }^{1} A_{1}$ & $V$-mix & 7.88 & $z$ & 0.3252 & 113.6843 & 66.9987 & 70.1911 & 250.8741 \\
\hline $8{ }^{1} B_{2}$ & $5 d_{x z}-R$ & 7.93 & $y$ & 0.0127 & 184.2486 & 67.3410 & 173.6527 & 425.2423 \\
\hline $9{ }^{1} A_{1}$ & $5 d_{x y}-R$ & 7.99 & $z$ & 0.0814 & 161.1558 & 144.0513 & 66.2029 & 371.4099 \\
\hline $12{ }^{1} B_{1}$ & $4 d^{\prime}-R$ & 8.02 & $x$ & 0.0001 & 105.1475 & 189.7472 & 184.0334 & 478.9280 \\
\hline $9^{1} \mathrm{~B}_{2}$ & $4 d_{x y}^{\prime}-R$ & 8.14 & $y$ & 0.0445 & 198.4062 & 188.6370 & 71.1856 & 458.2288 \\
\hline $10{ }^{1} A_{1}$ & $4 d_{x z}^{\prime}-R$ & 8.20 & $z$ & 0.0758 & 195.4445 & 73.8492 & 177.7282 & 447.0220 \\
\hline $10{ }^{1} B_{2}$ & $V$-mix & 8.25 & $y$ & 0.2553 & 69.5117 & 56.0848 & 33.4018 & 158.9982 \\
\hline $11{ }^{1} A_{1}$ & $5^{\prime} p_{x}-R$ & 8.32 & $z$ & 0.0014 & 398.9488 & 141.0667 & 139.6109 & 679.6265 \\
\hline $1{ }^{3} B_{2}$ & V & 4.58 & & & 26.7894 & 20.9241 & 19.4356 & 67.1491 \\
\hline $1{ }^{3} A_{2}$ & $3 s-R$ & 5.08 & & & 38.9417 & 31.5646 & 42.5046 & 113.0110 \\
\hline $1{ }^{3} A_{1}$ & V & 5.60 & & & 27.3228 & 22.2646 & 18.8990 & 68.4864 \\
\hline $1{ }^{3} B_{1}$ & $3 p_{y}-R$ & 5.82 & & & 40.0917 & 67.9226 & 34.3289 & 142.3432 \\
\hline
\end{tabular}

${ }^{a} V$ and $R$ denote, respectively, the valence and Rydberg excited state. For details see the text.

${ }^{\mathrm{b}}$ Transition energy.

${ }^{c}$ Polarization direction of the transition moment.

$\rightarrow 3 p_{x}, \pi^{*}, 3 d_{x z}$ ), $2{ }^{1} B_{2}$ (valence $1 a_{2} \rightarrow \pi^{*}, 3 p_{x}, 3 d_{x z}$ ) and $3{ }^{1} B_{2}\left(1 a_{2} \rightarrow 3 d_{x z}, \pi^{*}, 3 p_{x}\right)$ that are moderately separated in energy. Thus, an attempt to assign pure Rydberg or pure valence excitations in this case is meaningless. In our present calculations, we found that one valence transition configuration, $1 a_{2} \rightarrow \pi^{*}(18 \rightarrow 52)$, is included in all three ${ }^{1} B_{2}$ states, and its contributions (absolute value of the coefficient of configuration) to the three ${ }^{1} B_{2}$ states are $0.35,0.48$ and 0.53 , respectively. This further shows that the intensity of this energy region can be attributed mostly to valence excitation, and explains why Rydberg transitions $\left(1 a_{2} \rightarrow 3 p_{x}, \pi^{*}, 3 d_{x z}\right.$ and $\left.1 a_{2} \rightarrow 3 d_{x z}, \pi^{*}, 3 p_{x}\right)$ show strong intensity in this energy region. Our present calculated excitation energies, oscillator strengths, and second moments for the three ${ }^{1} B_{2}$ states are very similar to those by $\mathrm{CC} 3$ calculations. Assignment of the first ${ }^{1} B_{2}$ valence excitation in this energy region has been controversial in other theoretical studies. ${ }^{2,24,25,26,29}$ Although the $2{ }^{1} B_{2}$ state was calculated and assigned to be the valence excitation and was the major source of intensity for this energy region in CASPT2 $2^{22}$ and MRMP $^{25}$ studies, 
TABLE VI. SAC-CI results compared with the experimental excitation energies (in eV) and other theoretical results for pyrrole.

\begin{tabular}{|c|c|c|c|c|c|c|c|c|c|c|c|}
\hline \multirow[b]{2}{*}{ State } & \multirow{2}{*}{$\frac{\text { SAC }}{\text { Nature }^{\mathrm{a}}}$} & \multicolumn{2}{|c|}{$\mathrm{SAC}-\mathrm{CI}$} & \multirow[b]{2}{*}{ Expt. $^{c}$} & \multicolumn{2}{|c|}{$\mathrm{CASPT}^{\mathrm{d}}$} & \multirow{2}{*}{$\begin{array}{c}\text { MRMP }^{\mathrm{e}} \\
\Delta E\end{array}$} & \multicolumn{2}{|c|}{$\operatorname{ADC}(2)^{\mathrm{f}}$} & \multicolumn{2}{|c|}{$\mathrm{CC}^{\mathrm{g}}$} \\
\hline & & $f(r)$ & $\Delta E^{\mathrm{b}}$ & & $\Delta E$ & Nature & & $\Delta E$ & Nature & $\Delta E$ & Nature \\
\hline $1^{1} A_{2}$ & $3 s-R$ & Forbid & 5.11 & $\sim 5.22$ & 5.08 & $1^{1} A_{2}-3 s$ & 4.92 & 5.03 & $3 s\left({ }^{1} A_{2}\right)$ & 5.20 & $3 s\left({ }^{1} A_{2}\right)$ \\
\hline $1{ }^{1} B_{1}$ & $3 p_{y}-R$ & 0.0211 & 5.80 & $\sim 5.7$ & 5.85 & $1{ }^{1} B_{1}-3 p$ & 5.70 & 5.68 & $3 p\left({ }^{1} B_{1}\right)$ & 5.95 & $3 p_{y}\left({ }^{1} B_{1}\right)$ \\
\hline $2{ }^{1} A_{2}$ & $3 p_{z}-R$ & Forbid & 5.81 & & 5.83 & $2{ }^{1} A_{2}-3 p$ & 5.74 & 5.71 & $3 p\left({ }^{1} A_{2}\right)$ & 5.94 & $3 p_{z}\left({ }^{1} A_{2}\right)$ \\
\hline $1{ }^{1} B_{2}$ & $3 p_{x}-R / V$ & 0.0805 & 5.88 & $\sim 5.86$ & 5.78 & $1^{1} B_{2}-3 p$ & 5.87 & 5.86 & $3 p\left({ }^{1} B_{2}\right)$ & 6.04 & $3 p_{x}\left({ }^{1} B_{2}\right)$ \\
\hline $2{ }^{1} B_{1}$ & $3 s^{\prime}-R$ & 0.0005 & 6.05 & & 5.97 & $2{ }^{1} B_{1}-3 s^{\prime}$ & 5.81 & 5.77 & $3 s^{\prime}\left({ }^{1} B_{1}\right)$ & 6.12 & $3 s^{\prime}\left({ }^{1} B_{1}\right)$ \\
\hline $3{ }^{1} A_{2}$ & $3 d-R$ & Forbid & 6.38 & & 6.40 & $3{ }^{1} A_{2}-3 d$ & 6.45 & 6.25 & $3 d\left({ }^{1} A_{2}\right)$ & 6.51 & $3 d\left({ }^{1} A_{2}\right)$ \\
\hline $3{ }^{1} B_{1}$ & $3 d_{y z}-R$ & 0.0037 & 6.39 & $\sim 6.42$ & 6.42 & $3{ }^{1} B_{1}-3 d$ & 6.38 & 6.27 & $3 d\left({ }^{1} B_{1}\right)$ & 6.55 & $3 d_{y z}\left({ }^{1} B_{1}\right)$ \\
\hline $2{ }^{1} A_{1}$ & $V$ & 0.0002 & 6.41 & & 5.92 & $2{ }^{1} A_{1}-V$ & 5.98 & 6.66 & $V^{\prime}\left({ }^{1} A_{1}\right)$ & 6.37 & $V\left({ }^{1} A_{1}\right)$ \\
\hline $4{ }^{1} A_{2}$ & $3 d-R$ & Forbid & 6.44 & & 6.51 & $4{ }^{1} A_{2}-3 d$ & 6.44 & 6.37 & $3 d\left({ }^{1} A_{2}\right)$ & 6.57 & $3 d\left({ }^{1} A_{2}\right)$ \\
\hline $2{ }^{1} B_{2}$ & $V / 3 d_{x z}-R$ & 0.0475 & 6.48 & $6.2-6.5$ & 6.00 & $2{ }^{1} B_{2}-V$ & 6.51 & 6.48 & $3 d\left({ }^{1} B_{2}\right)$ & 6.57 & $V\left({ }^{1} B_{2}\right)$ \\
\hline $3{ }^{1} A_{1}$ & $3 d_{x y}-R$ & 0.0000 & 6.64 & & 6.54 & $3{ }^{1} A_{1}-3 d$ & 6.52 & 6.54 & $3 d\left({ }^{1} A_{1}\right)$ & 6.87 & $3 d_{x y}\left({ }^{1} A_{1}\right)$ \\
\hline $4{ }^{1} B_{1}$ & $3 p_{z}^{\prime}-R$ & 0.0266 & 6.68 & $6.5-6.7$ & 6.62 & $4{ }^{1} B_{1}-3 p^{\prime}$ & 6.48 & 6.43 & $3 p^{\prime}\left({ }^{1} B_{1}\right)$ & 6.82 & $3 p_{z}^{\prime}\left({ }^{1} B_{1}\right)$ \\
\hline $5{ }^{1} A_{2}$ & $4 s-R$ & Forbid & 6.71 & & & & & 6.55 & $4 s\left({ }^{1} A_{2}\right)$ & 6.92 & $4 s\left({ }^{1} A_{2}\right)$ \\
\hline $3{ }^{1} B_{2}$ & $3 d_{x z}-R / V$ & 0.0789 & 6.76 & 6.78 & 6.53 & $3{ }^{1} B_{2}-3 d$ & 6.61 & 6.71 & $V\left({ }^{1} B_{2}\right)$ & 6.90 & $3 d_{x z}\left({ }^{1} B_{2}\right)$ \\
\hline & & & & & 7.31 & $6{ }^{1} A_{2}-3 d^{\prime}$ & 7.25 & & & 6.97 & $3 p_{y}^{\prime}\left({ }^{1} A_{2}\right) ?$ \\
\hline $4{ }^{1} A_{1}$ & $3 p_{x}^{\prime}-R$ & 0.0146 & 6.86 & $7.0-7.1$ & 6.65 & $4{ }^{1} A_{1}-3 p^{\prime}$ & 6.62 & 6.42 & $3 p^{\prime}\left({ }^{1} A_{1}\right)$ & 7.04 & $3 p_{x}^{\prime}\left({ }^{1} A_{1}\right)$ \\
\hline $5{ }^{1} B_{1}$ & $4 p_{y}-R$ & 0.0029 & 6.93 & $7.0-7.1$ & & & & 6.78 & $4 p\left({ }^{1} B_{1}\right)$ & 7.17 & $4 p_{y}\left({ }^{1} B_{1}\right)$ \\
\hline $6{ }^{1} A_{2}$ & $4 p_{z}-R$ & Forbid & 6.95 & & 6.77 & $5^{1} A_{2}-3 d^{\prime}$ & 6.70 & 6.78 & $4 p\left({ }^{1} A_{2}\right)$ & 7.16 & $4 p_{z}\left({ }^{1} A_{2}\right)$ \\
\hline $4{ }^{1} B_{2}$ & $4 p_{x}-R$ & 0.0020 & 6.99 & $7.0-7.1$ & & & & 6.88 & $4 p\left({ }^{1} B_{2}\right)$ & 7.20 & $4 p_{x}\left({ }^{1} B_{2}\right)$ \\
\hline $7{ }^{1} A_{2}$ & $4 d-R$ & Forbid & 7.11 & & & & & 6.98 & $4 d\left({ }^{1} A_{2}\right)$ & 7.34 & $4 d\left({ }^{1} A_{2}\right)$ \\
\hline $8{ }^{1} A_{2}$ & $4 d-R$ & Forbid & 7.13 & & & & & 6.99 & $4 d\left({ }^{1} A_{2}\right)$ & 7.36 & $4 d\left({ }^{1} A_{2}\right)$ \\
\hline $6{ }^{1} B_{1}$ & $4 d_{y z}-R$ & 0.0001 & 7.14 & & & & & 7.00 & $4 d\left({ }^{1} B_{1}\right)$ & 7.37 & $4 d_{y z}\left({ }^{1} B_{1}\right)$ \\
\hline $5{ }^{1} A_{1}$ & $4 d_{x y}-R$ & 0.0041 & 7.26 & $\sim 7.35$ & & & & 7.12 & $4 d\left({ }^{1} A_{1}\right)$ & 7.48 & $4 d_{x y}\left({ }^{1} A_{1}\right)$ \\
\hline $7{ }^{1} B_{1}$ & $3 d^{\prime}-R$ & 0.0018 & 7.26 & & 7.39 & $6{ }^{1} B_{1}-3 d^{\prime}$ & 7.23 & 6.97 & $3 d^{\prime}\left({ }^{1} B_{1}\right)$ & 7.41 & $3 d^{\prime}\left({ }^{1} B_{1}\right)$ \\
\hline $5{ }^{1} B_{2}$ & $4 d_{x z}-R$ & 0.0119 & 7.26 & $\sim 7.4$ & 7.72 & $5{ }^{1} B_{2}-4 d$ & & 7.14 & $4 d\left({ }^{1} B_{2}\right)$ & 7.48 & $4 d_{x z}\left({ }^{1} B_{2}\right)$ \\
\hline $9{ }^{1} A_{2}$ & $5 s-R$ & Forbid & 7.27 & & & & & & & & \\
\hline $8{ }^{1} B_{1}$ & $3 d^{\prime}-R$ & 0.0092 & 7.34 & & 7.32 & $5{ }^{1} B_{1}-3 d^{\prime}$ & 7.14 & 7.09 & $3 d^{\prime}\left({ }^{1} B_{1}\right)$ & 7.47 & $3 d^{\prime}\left({ }^{1} B_{1}\right)$ \\
\hline $10{ }^{1} A_{2}$ & $5 p_{z}-R$ & Forbid & 7.44 & & & & & & & & \\
\hline $9{ }^{1} B_{1}$ & $5 p_{y}-R$ & 0.0048 & 7.44 & $\sim 7.54$ & & & & & & $\ldots$ & \\
\hline $6{ }^{1} B_{2}$ & $5 p_{x}-R$ & 0.0020 & 7.45 & & & & & & & & ore Ryds \\
\hline $6{ }^{1} A_{1}$ & $3 d_{x z}^{\prime}-R$ & 0.0679 & 7.49 & & 7.36 & $5^{1} A_{1}-3 d^{\prime}$ & 7.20 & 7.23 & $3 d^{\prime}\left({ }^{1} A_{1}\right)$ & $\ldots$ & \\
\hline $7{ }^{1} B_{2}$ & $3 d_{x y}^{\prime}-R$ & 0.0052 & 7.55 & $\sim 7.7$ & 7.43 & $4{ }^{1} B_{2}-3 d^{\prime}$ & 7.36 & 7.26 & $3 d^{\prime}\left({ }^{1} B_{2}\right)$ & & \\
\hline $10{ }^{1} B_{1}$ & $4 s^{\prime}-R$ & 0.0000 & 7.63 & & & & & 7.24 & $4 s^{\prime}\left({ }^{1} B_{1}\right)$ & & \\
\hline $11{ }^{1} B_{1}$ & $5 d_{y z}-R$ & 0.0002 & 7.75 & $\sim 7.85$ & & & & & & & \\
\hline $7^{1} A_{1}$ & $4^{\prime} p_{x}-R$ & 0.0020 & 7.83 & $\sim 7.95$ & & & & & & & \\
\hline $8{ }^{1} A_{1}$ & $V$-mix & 0.3252 & 7.88 & $7.5-8$ & 7.46 & $6{ }^{1} A_{1}-V$ & 7.48 & 7.75 & $V\left({ }^{1} A_{1}\right)$ & 7.91 & $V\left({ }^{1} A_{1}\right)$ \\
\hline $8{ }^{1} B_{2}$ & $5 d_{x z}-R$ & 0.0127 & 7.93 & $\sim 8.0$ & & & & & & & \\
\hline $9^{1} A_{1}$ & $5 d_{x y}-R$ & 0.0814 & 7.99 & $\sim 8.1$ & & & & & & $\cdots$ & \\
\hline $12{ }^{1} B_{1}$ & $4 d^{\prime}-R$ & 0.0001 & 8.02 & & & & & & & & ore Ryds \\
\hline $9{ }^{1} B_{2}$ & $4 d_{x y}^{\prime}-R$ & 0.0445 & 8.14 & & & & & & & $\ldots$ & \\
\hline $10{ }^{1} A_{1}$ & $4 d_{x y}^{\prime \prime}-R$ & 0.0758 & 8.20 & & 7.88 & $7{ }^{1} A_{1}-4 d^{\prime}$ & & & & & \\
\hline $10{ }^{1} B_{2}$ & $V$-mix & 0.2553 & 8.25 & & & & & & & 8.31 & $V\left({ }^{1} B_{2}\right)$ \\
\hline $11{ }^{1} A_{1}$ & $5^{\prime} p_{x}-R$ & 0.0014 & 8.32 & & & & & & & & \\
\hline $1^{3} B_{2}$ & $V$ & & 4.58 & 4.2 & 4.21 & & & 4.59 & & & \\
\hline $1{ }^{3} A_{2}$ & $3 s-R$ & & 5.08 & & 5.04 & & & 5.00 & & & \\
\hline $1^{3} A_{1}$ & V & & 5.60 & 5.10 & 5.16 & & & 5.55 & & & \\
\hline $1{ }^{3} B_{1}$ & $3 p_{y}-R$ & & 5.82 & & 5.82 & & & 5.63 & & & \\
\hline
\end{tabular}

${ }^{a} V$ and $R$ denote, respectively, valence and Rydberg excited state. For details see the text.

${ }^{\mathrm{b}}$ Transition energy.

'Experimental data are taken from Refs. 3, 7, 9, and 22, and references therein. Those for valence excitations are chosen as the bands' maximum; those for Rydberg transitions are $0-0$ excitation energies.

${ }^{\mathrm{d}}$ Reference 21 .

${ }^{\mathrm{e}}$ Reference 23.

${ }^{\mathrm{f}}$ Reference 24 .

${ }^{\mathrm{g}}$ Reference 25 .

the difference in the calculated vertical excitation energy of the $2{ }^{1} B_{2}$ state between CASPT2 and MRMP studies is as large as $0.51 \mathrm{eV}$. In recent $\mathrm{ADC}(2)$ studies, ${ }^{26}$ however, the first ${ }^{1} B_{2}$ valence excitation was suggested to be outside this energy region, and the intensity for this region was ascribed mostly to the $1 a_{2} \rightarrow 3 p\left(1{ }^{1} B_{2}\right)$ Rydberg transition.

A weak singlet excited state has been observed at about
$5.22 \mathrm{eV}$ and assigned to one ${ }^{1} A_{1}$ state in the VUV absorption spectrum by Mullen and Orloff. ${ }^{3}$ However, this assignment was disputed by Robin ${ }^{15}$ and experimentally assigned to a Rydberg state. The present calculations compute the $1{ }^{1} A_{2}$ state with $1 a_{2} \rightarrow 3 s$ nature at $5.11 \mathrm{eV}$, which supports the notion that the lowest singlet state might be due to the vibrational structure of the dipole-forbidden $1 a_{2} \rightarrow 3 s$ Rydberg 


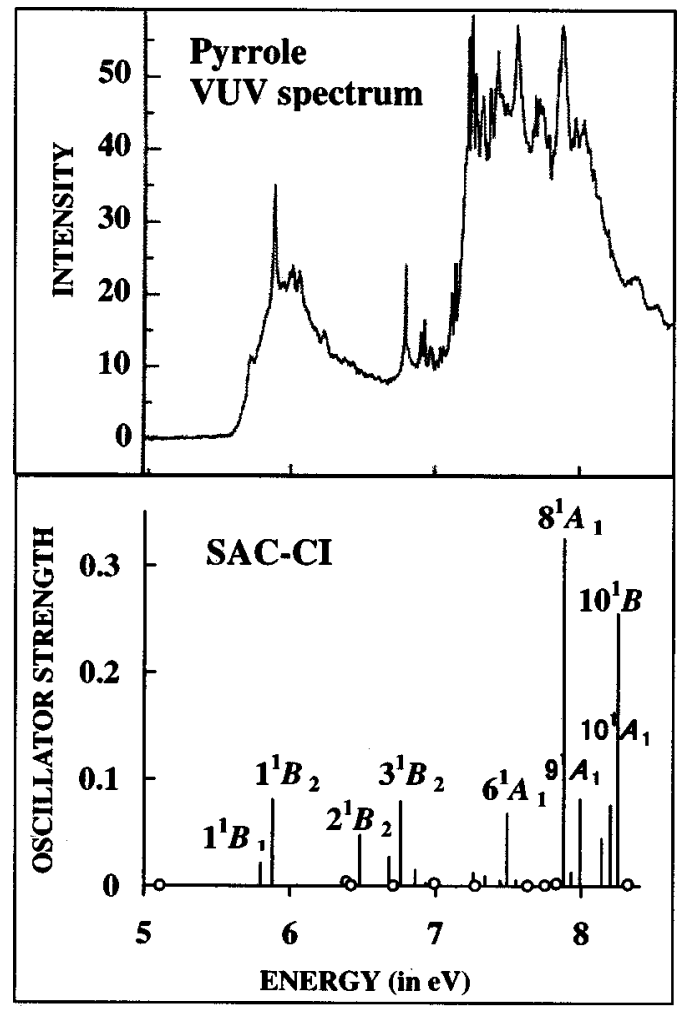

FIG. 4. VUV spectrum and SAC-CI theoretical spectrum of pyrrole.

transition, since the present calculations, together with previous high-level calculations, ${ }^{22,26,29}$ show that there is no other singlet state in this energy region.

Next, we discuss the valence excitation, $V^{\prime}\left({ }^{1} A_{1}\right)$, which is expected to occur in the first band. As we discussed for the $2{ }^{1} A_{1}$ state of furan, a similar situation occurs in the $2{ }^{1} A_{1}$ state of pyrrole. Our present calculations give the $2{ }^{1} A_{1}$ state at $6.41 \mathrm{eV}$ with an oscillator strength of 0.0002 . Its second moment $\left\langle r^{2}\right\rangle$ is 73.3451 a.u. ${ }^{2}$, which clearly characterizes it as a valence excited state, and its nature is a mixture of $\pi_{2}-\pi^{*}(17 \rightarrow 52)$ and $\pi_{3}-\pi^{*}$ transitions $(18 \rightarrow 53)$ and $(18 \rightarrow 66)$. Double excitation makes a rather small contribution. $\mathrm{CC} 3$ computed it to be $6.37 \mathrm{eV}$ with an oscillator strength of 0.001 . $\operatorname{ADC}(2)$ computed this state at $6.66 \mathrm{eV}$ with an oscillator strength of 0.017 . CASPT2 computed the $2{ }^{1} A_{1}$ state at $5.92 \mathrm{eV}$ with an intensity of 0.0195 , and its nature was reported to be a mixture of the single excitation
$2 b_{1} \rightarrow 3 b_{1}$ and $1 a_{2} \rightarrow 2 a_{2}$ configurations in combination with a large fraction $(25 \%)$ of double excitations $\left(1 a_{2}\right)^{2}$ $\rightarrow\left(3 b_{1}\right)^{2}$. MRMP computed this state at $5.98 \mathrm{eV}$ with an intensity of 0.0098 , and its nature was almost the same as that of CASPT2. Note that here there is again a discrepancy between SAC-CI, CC3, and CASPT2, MRMP results. However, in the case of thiophene, which will be discussed in a later paper, ${ }^{43}$ the $2{ }^{1} A_{1}$ state (lowest excited state) has been well characterized to be predominantly single excitation by both SAC-CI and CASPT2 with regard to both excitation energy and oscillator strength, in good accordance with the experimental data of the VUV and magnetic circular dichroism spectra. Generally speaking, the SAC-CI SD-R method used here, together with the CCSD/CC3 and EOM-CCSD(T) methods, is best applied to states that are largely single excitation in nature, and may give a relatively high excitation energy estimate for the state of significant doubly excited character. Therefore, the doubly excited characters of the $2{ }^{1} A_{1}$ states of this series of five-membered ring compounds deserve to be further investigated by the SAC-CI general-R method, ${ }^{44}$ which is suitable for two- and many-electron processes.

In the VUV spectrum of Mullen and Orloff, ${ }^{3}$ absorption peaks are observed at around 5.71 and $5.88 \mathrm{eV}$, and a question arises as to whether these two absorption peaks represent two separate electronic transitions or one transition with an associated vibrational structure. In the later UV spectrum of Bavia et al., ${ }^{7}$ absorption peaks were reported at around 5.82 and $5.86 \mathrm{eV}$, however, an obvious absorption peak at around $5.7 \mathrm{eV}$ was ignored. In the latest VUV spectrum of Palmer et al. ${ }^{24}$ absorption peaks were reported at around 5.698 and $5.861 \mathrm{eV}$ and assigned to the $1{ }^{1} B_{2}\left(1 a_{2} \rightarrow 3 p^{\pi}\right)$ and $1{ }^{1} B_{1}\left(1 a_{2} \rightarrow 3 p_{y}\right)$ states, respectively. The $5.861 \mathrm{eV}$ transition is the most intense and shows obvious vibrational structures in the first band. Other nearby peaks, e.g., 5.82 and $5.88 \mathrm{eV}$, are interpreted as part of the vibrational structure of this transition. Palmer et al. ${ }^{24}$ by comparing this with the envelope of the first band in PES, ${ }^{5}$ confirmed that the 5.861 $\mathrm{eV}$ absorption arises from the excitation of a $1 a_{2} \rightarrow 3 p$ Rydberg state associated with $\mathrm{IP}_{1}(8.21 \mathrm{eV})$. Our present calculations support this general experimental assignment, but the calculated excitation energy orders and oscillator strengths prefer the assignment of the $5.861 \mathrm{eV}$ transition to $1{ }^{1} B_{2}\left(1 a_{2} \rightarrow 3 p_{x}, \pi^{*}\right)$ and the $5.698 \mathrm{eV}$ transition to

TABLE VII. Comparison of electronic transition mechanisms in pyrrole and furan.

\begin{tabular}{|c|c|c|c|c|c|c|c|c|c|c|c|}
\hline \multicolumn{6}{|c|}{ Pyrrole } & \multicolumn{6}{|c|}{ Furan } \\
\hline State & Nature & $\Delta E$ & $f(r)$ & $\mathrm{SM}^{\mathrm{a}}$ & $\mathrm{MC}^{\mathrm{b}}$ & State & Nature & $\Delta E$ & $f(r)$ & SM & $\mathrm{MC}$ \\
\hline $1{ }^{1} A_{1}$ & Ground & 0 & & -65.3 & & $1{ }^{1} A_{1}$ & Ground & 0 & & -64.7 & \\
\hline $1{ }^{1} B_{2}$ & $3 p_{x}-R \sim V$ & 5.88 & 0.081 & -132.1 & $1 a_{2}-3 p_{x}, 1 a_{2}-\pi^{*}$ & $1{ }^{1} B_{2}$ & $V$-mix & 6.40 & 0.185 & -92.5 & $1 a_{2}-\pi^{*}$ \\
\hline $2{ }^{1} A_{1}$ & $V$ & 6.41 & 0.000 & -73.3 & $2 b_{1}-\pi^{*}, 1 a_{2}-\pi^{*}$ & $2{ }^{1} A_{1}$ & V & 6.79 & 0.000 & -69.0 & $2 b_{1}-\pi^{*}, 1 a_{2}-\pi^{*}$ \\
\hline $2{ }^{1} B_{2}$ & $V-3 d_{x z}-R$ & 6.48 & 0.048 & -146.3 & $1 a_{2}-\pi^{*}, 1 a_{2}-3 d_{x z}$ & $2{ }^{1} B_{2}$ & $3 p_{x}-R$ & 6.82 & 0.016 & -129.5 & $1 a_{2}-3 p_{x}$ \\
\hline $3{ }^{1} B_{2}$ & $3 d_{x z}-R \sim V$ & 6.76 & 0.079 & -224.7 & $1 a_{2}-3 d_{x z}, 1 a_{2}-\pi$ & $3{ }^{1} B_{2}$ & $3 d_{x z}-R$ & 7.51 & 0.019 & -245.7 & $1 a_{2}-3 d_{x z}$ \\
\hline $8{ }^{1} A_{1}$ & $V$-mix & 7.88 & 0.325 & -250.9 & $1 a_{2}-\pi^{*}, 2 b_{1}-\pi^{*}$ & $6{ }^{1} A_{1}$ & $V$-mix & 8.34 & 0.483 & -97.5 & $1 a_{2}-\pi^{*}, 2 b_{1}-\pi^{*}$ \\
\hline $10^{1} B_{2}$ & $V$-mix & 8.25 & 0.255 & -159.0 & $2 b_{1}-\pi^{*}$ & $9{ }^{1} B_{2}$ & $V$-mix & 9.08 & 0.166 & -130.4 & $2 b_{1}-\pi^{*}$ \\
\hline
\end{tabular}

${ }^{a} \mathrm{SM}$ denotes second moment.

${ }^{\mathrm{b}} \mathrm{MC}$ denotes main configuration. 
$1{ }^{1} B_{1}\left(1 a_{2} \rightarrow 3 p_{y}\right)$, which is opposite the assignments of Palmer et al. ${ }^{22}$ Except for CASPT2, all previous theoretical studies, i.e., CC3, MRMP, and ADC(2), gave similar trends for excitation energy orders and oscillator strengths to our present results, however, all of them followed Bavia et al. ${ }^{7}$ and ignored the $5.7 \mathrm{eV}$ peak, and assigned the $5.861 \mathrm{eV}$ transition to $1{ }^{1} B_{2}\left(1 a_{2} \rightarrow 3 p_{x}\right)$ and the 5.82 or $5.88 \mathrm{eV}$ transition instead of the $5.7 \mathrm{eV}$ transition to $1{ }^{1} B_{1}\left(1 a_{2} \rightarrow 3 p_{y}\right)$.

The next peak in the VUV spectrum is observed at around $6.2 \mathrm{eV}$. This peak was assigned experimentally to the $2 b_{1} \rightarrow 3 s$ Rydberg transition by Derrick et al. ${ }^{5}$ On the other hand, this peak, together with other peaks at slightly higher energies, was assigned experimentally to a vibrational structure of the $1 a_{2} \rightarrow 3 p_{x}$ Rydberg state by Bavia et al. ${ }^{7}$ Our present calculations support an alternative assignment for this group of peaks, and associates them with the $V\left(2{ }^{1} B_{2}\right)$ state calculated at $6.48 \mathrm{eV}$ and its vibrational structure. Additionally, the $1 a_{2} \rightarrow 3 d_{y z}\left(3{ }^{1} B_{1}\right)$ Rydberg states, etc., are also predicted to be in this region. Note that the absorption peaks were reported at around $6.42 \mathrm{eV}$ and assigned to the $1 a_{2} \rightarrow 3 d$ Rydberg state in the UV spectrum of Bavia et al. ${ }^{7}$

\section{The second weak central band (6.6-7.1 eV) in the VUV spectrum}

A relatively intense absorption peak was observed at around $6.78 \mathrm{eV}$ in the VUV spectrum by Palmer et al., ${ }^{24}$ and assigned to the $1 a_{2} \rightarrow 3 d$ Rydberg transition instead of $1 a_{2}$ $\rightarrow 4 s,{ }^{7}$ which is dipole forbidden. Two other weaker bands at 7.021 and $7.044 \mathrm{eV}$ were assigned to the excitations of the $2 b_{1} \rightarrow 3 p$ series of Rydberg transitions. ${ }^{24}$ Our present calculations support the above-given general assignments of this energy region. The $3{ }^{1} B_{2}$ state $\left(1 a_{2} \rightarrow 3 d_{x z}\right)$ is computed at $6.76 \mathrm{eV}$ with an oscillator strength of 0.0789 , which is the most intense absorption peak in this energy region and hence is reasonably associated with the absorption peak at around $6.78 \mathrm{eV}$ in the VUV spectrum. It is easy to understand why this Rydberg transition is intense via the above-mentioned hybrid model. It is mixed with valence transition and hence it "borrows" the intensity from the valence transition. Most of the intensity of the first and second bands can be attributed to the same valence transition. Additionally, the $2 b_{1} \rightarrow 3 p$ series of Rydberg states are also predicted in this region, and the $1 a_{2} \rightarrow 4 p$ series of Rydberg states are predicted at the high-energy tail of this energy region. Note that CC3 calculations predicted very similar results in this energy region and also supported the assignment. However, CASPT2 and MRMP assigned the $6.78 \mathrm{eV}$ peak to the $2 b_{1} \rightarrow 3 p$ series of Rydberg transitions according to their calculations.

\section{The third strong intense and broad band (7.1-8.2 eV) in the VUV spectrum}

A strong intense and broad absorption superimposed with a few sharp peaks was observed at 7.1-8.2 eV in the VUV spectrum. Our present calculations computed the $8{ }^{1} A_{1}$ state at $7.88 \mathrm{eV}$ with an oscillator strength of 0.3252 and the $10{ }^{1} B_{2}$ state at $8.25 \mathrm{eV}$ with an oscillator strength of 0.2553 . The nature of the $8{ }^{1} A_{1}$ state is a strong mixture of the $\pi \rightarrow \pi^{*}$ transitions $(18 \longrightarrow 53)$ and $(17 \rightarrow 52)$ with the Rydberg transitions $(17 \rightarrow 26)$ and $(18 \rightarrow 38)$, etc., which are respon- sible for the rather large second moment $\left(250.9\right.$ a.u. $\left.{ }^{2}\right)$ of this state. The nature of the $10{ }^{1} B_{2}$ state is of strong mixture of $\pi \rightarrow \pi^{*}$ transitions $(17 \rightarrow 53),(17 \rightarrow 66)$, and $(18 \rightarrow 52)$ with Rydberg transition $(17 \rightarrow 27)$, etc., which is responsible for the large second moment (159.0 a.u. $\left.{ }^{2}\right)$ of this state. The strong mixture of $\pi \rightarrow \pi^{*}$ excitations with Rydberg excitations reflects the strong interactions between valence and Rydberg excited states. Note that these interactions in pyrrole are stronger than those in furan. The two states mentioned previously are defined as energetically high valence states of $A_{1}$ and $B_{2}$ symmetries, respectively, considering oscillator strengths, main configurations, and second moments. Our present calculations show that a significant portion of the electronic absorption intensity in this region is due to the two energetically high valence excitations mentioned previously. We also predict that the $1 a_{2} \rightarrow 4 d$ series, $1 a_{2} \rightarrow 5 p$ series, $2 b_{1} \rightarrow 3 d$ series, $1 a_{2} \rightarrow 5 d$ series, $2 b_{1} \rightarrow 4 d$ series, etc., Rydberg states are located in this energy region, and some of them have relatively large oscillator strengths. There are so many Rydberg states in only a $1 \mathrm{eV}$ range, and therefore it is difficult to make an accurate assignment of Rydberg transitions in this energy region. For vibrational and geometrical relaxations, Rydberg transitions can easily overlap, and thus the absorption in this region becomes very complicated. However, we believe that it is reasonable to associate the valence and Rydberg excitations mentioned previously to the fine structure in the region $7.1-8.2 \mathrm{eV}$.

\section{Triplet excited states}

The two lowest triplet transitions of pyrrole have been identified by the electron energy-loss (EEL) ${ }^{8-10,24}$ spectrum at 4.2 and $5.1 \mathrm{eV}$, which are assigned to the ${ }^{3} B_{2}$ and ${ }^{3} A_{1}$ valence transitions, respectively. Our present calculations computed the $1{ }^{3} B_{2}$ state at $4.58 \mathrm{eV}$ with a second moment of 67.1 a.u. ${ }^{2}$, and the $1^{3} A_{1}$ state at $5.60 \mathrm{eV}$ with a second moment of 68.5 a.u. ${ }^{2}$ The second moments of these two states (listed in Table V) clearly characterize them as valence excitations. The main configurations of $1^{3} B_{2}$ are $(18 \rightarrow 52)$, $(18 \rightarrow 62),(18 \rightarrow 59),(18 \rightarrow 64)$, etc., and those of $1^{3} A_{1}$ are $(17 \rightarrow 52),(18 \rightarrow 66),(18 \rightarrow 53),(17 \rightarrow 62)$, etc. In the same energy region, we found two Rydberg excited states $1^{3} A_{2} 1{ }^{3} A_{1}$ and $1{ }^{3} B_{2}$, which are given at 5.08 and $5.82 \mathrm{eV}$, respectively.

\section{Comparison of furan and pyrrole}

Comparing the present calculations of furan with those of pyrrole, it is obvious that a similar electronic excitation mechanism occurs in both molecules. There are two valence excitations in $B_{2}$ and $A_{1}$ symmetry, respectively, which are responsible for most of the intensity of the electronic absorption bands, and hence span the general profiles of the VUV spectra in the energy region up to around $8.2 \mathrm{eV}$ in pyrrole and $8.8 \mathrm{eV}$ in furan. From the main configurations listed in Table VII, we can see that both $1 a_{2}$ and $2 b_{1}$ series $\pi \rightarrow \pi^{*}$ valence transition configurations contribute to the two valence excitations of $A_{1}$ symmetry in both molecules. However, the $1 a_{2}$ series $\pi \rightarrow \pi^{*}$ valence transition configurations predominantly contribute to the lowest valence excitation of 
$B_{2}$ symmetry and the $2 b_{1}$ series $\pi \rightarrow \pi^{*}$ predominantly contribute to the higher-energy valence excitation of $B_{2}$ symmetry in both molecules. At the same time, these valence excitations are more or less influenced by low-lying Rydberg transitions. In the case of pyrrole, due to the lower first ionization potential (1IPs), the Rydberg states are expected to be in the lower energy region, which is proven by our present calculations. In furan, the lowest excited state is the valence excitation of $B_{2}$ symmetry except for the dipole-forbidden $3 s$ Rydberg transition, whereas in pyrrole the energies of the $3 p$ series Rydberg transitions are lower than that of the lowest valence excitation of $B_{2}$ symmetry, which gives rise to stronger interactions between valence and Rydberg excitations than in furan. It is also clear from the second moments of these states that the interactions between valence and Rydberg transitions in pyrrole are much stronger than those in furan, which is responsible for the more complicated VUV fine structure of pyrrole.

Comparing the present SAC-CI results with those obtained by the CCSD and CC3 methods, we find that the general trends are very similar, and the difference in excitation energies is very small, less than $0.1 \mathrm{eV}$ (maximum) for valence excitations and $0.25 \mathrm{eV}$ (maximum) for Rydberg transitions. These similar results are natural since the basic theories are quite similar and the difference lies only in computational methodology: the former uses perturbation selection schemes and the latter adopts integral-direct strategies. The present results, comparing with CCSD/CC3 results, show that the perturbation selection scheme in the SAC-CI SD-R method is very effective and reserves a high accuracy at the same time.

\section{CONCLUSIONS}

The SAC/SAC-CI theory was applied to furan and pyrrole to reinvestigate their electronic excited states in more detail, including the singlet and triplet valence and Rydberg excited states. The SAC/SAC-CI method with a sufficiently extended basis set and a large active space gave a quantitatively satisfactory result to make reliable assignments for the electron excitation spectrum of furan and pyrrole. The present results show good agreement with various experimental data measured by VUV and EEL spectroscopies. The mixing of valence and Rydberg states was stronger in pyrrole than in furan.

In the case of furan, four singlet valence $\pi-\pi^{*}$ excitations (two $A_{1}$ states and two $B_{2}$ states), which span the general profile of the VUV spectrum, agree well with the latest experimental data by Palmer et al. ${ }^{23}$ In particular, our present results strongly support the notion that valence excitation exists in the $8.7 \mathrm{eV}$ energy region. In addition, many Rydberg excitations were determined in the present calculations, and the corresponding assignments in this work should constitute a useful theoretical reference for experimental chemists in this field.

In the case of pyrrole, 43 low-lying singlet excited states were obtained theoretically and reliable assignments for the VUV spectrum up to $8.2 \mathrm{eV}$ were made using the calculated energies and oscillator strengths. Valence and Rydberg excited states were discussed separately in detail. For the first intense band and second weak band of the VUV spectrum, our present calculated results show a strong mixture of valence and Rydberg excitations in the first three ${ }^{1} B_{2}$ excited states. For the third strong and broad band, the present calculations give two valence excited states, $8{ }^{1} A_{1}$ and $10^{1} B_{2}$, which are strongly influenced by Rydberg transitions. These valence excitations, together with Rydberg excitations, span the general profile of the VUV spectrum observed by Palmer et al. $^{24}$

\section{ACKNOWLEDGMENTS}

This research was supported by a Grant-in-Aid for Scientific Research from the Ministry of Education, Science, Culture and Sports. The authors are grateful to K. Toyota for helpful discussions on this subject.

${ }^{1}$ W. C. Price and A. D. Walsh, Proc. R. Soc. London, Ser. A 179, 201 (1941).

${ }^{2}$ L. W. Pickett, N. J. Hoeflich, and T.-C. Liu, J. Am. Chem. Soc. 73, 4865 (1951).

${ }^{3}$ P. A. Mullen and M. K. Orloff, J. Chem. Phys. 51, 2276 (1969).

${ }^{4}$ J. H. D. Eland, J. Mass Spectrom. Ion Phys. 2, 471 (1969).

${ }^{5}$ P. J. Derrick, L. Asbrink, O. Edqvist, B.-O. Jonsson, and E. Lindholm, Int. J. Mass Spectrom. Ion Phys. 6, 161 (1971).

${ }^{6}$ P. J. Derrick, L. Asbrink, O. Edqvist, and E. Lindholm, Spectrochim. Acta A 27, 2525 (1971).

${ }^{7}$ M. Bavia, F. Bertinelli, C. Taliani, and C. Zauli, Mol. Phys. 31, 479 (1976).

${ }^{8}$ W. M. Flicker, O. A. Mosher, and A. Kuppermann, J. Chem. Phys. 64, 1315 (1976).

${ }^{9}$ W. M. Flicker, O. A. Mosher, and A. Kuppermann, Chem. Phys. Lett. 38, 489 (1976).

${ }^{10}$ E. H. Van Veen, Chem. Phys. Lett. 41, 535 (1976).

${ }^{11}$ B. Norden, R. Hakansson, P. B. Pedersen, and E. W. Thulstrup, Chem. Phys. 33, 355 (1978).

${ }^{12}$ C. D. Cooper, A. D. Williamson, J. C. Miller, and R. N. Compton, J. Chem. Phys. 73, 1527 (1980).

${ }^{13}$ J. L. Roebber, D. P. Gerrity, R. Hemley, and V. Vaida, Chem. Phys. Lett. 75, 104 (1980).

${ }^{14}$ L. Nyulaszi, J. Mol. Struct. 273, 133 (1992).

${ }^{15}$ M. B. Robin, Higher Excited States of Polyatomic Molecules (Academic, New York, 1975), Vol. II.

${ }^{16}$ K. Tanaka, T. Nomura, T. Noro, H. Tatewaki, T. Takada, H. Kashiwagi, F. Sasaki, and K. Ohno, J. Chem. Phys. 67, 5738 (1977).

${ }^{17}$ W. Butscher and K.-H. Thunemann, Chem. Phys. Lett. 57, 224 (1978).

${ }^{18}$ K.-H. Thunemann, R. J. Buenker, and W. Butscher, J. Chem. Phys. 47, $313(1980)$.

${ }^{19}$ D. C. Rawlings and E. R. Davidson, Chem. Phys. Lett. 98, 424 (1983).

${ }^{20}$ D. C. Rawlings, E. R. Davidson, and M. Gouterman, Int. J. Quantum Chem. 26, 237 (1984).

${ }^{21}$ H. Nakatsuji, O. Kitao, and T. Yonezawa, J. Chem. Phys. 83, 723 (1985).

${ }^{22}$ L. Serrano-Andres, M. Merchan, I. Nebot-Gil, B. O. Roos, and M. Fulscher, J. Am. Chem. Soc. 115, 6184 (1993).

${ }^{23}$ M. H. Palmer, I. C. Walker, C. C. Ballard, and M. F. Guest, Chem. Phys. 192, 111 (1995)

${ }^{24}$ M. H. Palmer, I. C. Walker, and M. F. Guest, Chem. Phys. 238, 179 (1998).

${ }^{25}$ H. Nakano, T. Tsuneda, T. Hashimoto, and K. Hirao, J. Chem. Phys. 104, 2312 (1996).

${ }^{26}$ A. B. Trofimov and J. Schirmer, Chem. Phys. 214, 153 (1997).

${ }^{27}$ A. B. Trofimov and J. Schirmer, Chem. Phys. 224, 175 (1997).

${ }^{28}$ O. Christiansen and P. Jørgensen, J. Am. Chem. Soc. 120, 3423 (1998).

${ }^{29}$ O. Christiansen, J. Gauss, J. Stanton, and P. Jørgensen, J. Chem. Phys. 111, 525 (1999).

${ }^{30}$ Landolt-Bornstein, Numerical Data and Functional Relationships in Science and Technology, New Series, Group II: Atomic and Molecular Physics, Structure Data of Free Polyatomic Molecules, Vol. 7, edited by J. H. Callomon, E. Hirota, K. Kuchitsu, W. J. Lafferty, A. G. Maki, and C. S. Pote (Springer, Berlin, 1976). 
${ }^{31}$ R. A. Kendall, T. H. Dunning, Jr., and R. J. Harrison, J. Chem. Phys. 96, 6796 (1992)

${ }^{32}$ T. H. Dunning, Jr., J. Chem. Phys. 90, 1007 (1989).

${ }^{33}$ K. Kaufmann, W. Baumeister, and M. Jungen, J. Phys. B 22, 2223 (1989).

${ }^{34}$ GaUSSiAn 98 (Revision A.1), M. J. Frisch, G. W. Trucks, H. B. Schlegel, G. E. Scuseria, M. A. Robb, J. R. Cheeseman, V. G. Zakrzewski, J. A. Montgomery, R. E. Stratmann, J. C. Burant, S. Dapprich, J. M. Millam, A. D. Daniels, K. N. Kudin, M. C. Strain, O. Farkas, J. Tomasi, V. Barone, M. Cossi, R. Cammi, B. Mennucci, C. Pomelli, C. Adamo, S. Clifford, J. Ochterski, G. A. Petersson, P. Y. Ayala, Q. Cui, K. Morokuma, D. K. Malick, A. D. Rabuck, K. Raghavachari, J. B. Foresman, J. Cioslowski, J. V. Ortiz, B. B. Stefanov, G. Liu, A. Liashenko, P. Piskorz, I. Komaromi, R. Gomperts, R. L. Martin, D. J. Fox, T. Keith, M. A. Al-Laham, C. Y. Peng, A. Nanayakkara, C. Gonzalez, M. Challacombe, P. M. W. Gill, B. G. Johnson, W. Chen, M. W. Wong, J. L. Andres, M. Head-Gordon, E. S. Replogle, and J. A. Pople, Gaussian, Inc., Pittsburgh, PA (1998).
${ }^{35}$ H. Nakatsuji and K. Hirao, J. Chem. Phys. 68, 2053 (1978).

${ }^{36}$ H. Nakatsuji, Chem. Phys. Lett. 59, 362 (1978).

${ }^{37}$ H. Nakatsuji, Chem. Phys. Lett. 67, 329 (1979).

${ }^{38}$ H. Nakatsuji, Computational Chemistry: Reviews of Current Trends, edited by J. Leszczynski (World Scientific, Singapore, 1997), Vol. 2.

${ }^{39}$ K. Hirao and H. Nakatsuji, Chem. Phys. Lett. 79, 292 (1981).

${ }^{40}$ H. Nakatsuji, Chem. Phys. 75, 425 (1983).

${ }^{41}$ H. Nakatsuji, M. Hada, M. Ehara, J. Hasegawa, T. Nakajima, H. Nakai, O. Kitao, and K. Toyota, SAC/SAC-CI program system (SAC-CI96) for calculating ground, excited, ionized, and electron attached states and singlet to septet spin multiplicities.

${ }^{42}$ J. Wan, M. Ehara, M. Hada, and H. Nakatsuji, J. Chem. Phys. (to be published).

${ }^{43}$ J. Wan, M. Hada, M. Ehara, and H. Nakatsuji (unpublished).

${ }^{44}$ H. Nakatsuji, Chem. Phys. Lett. 59, 331 (1991). 